\title{
Investigating the stability of viscoelastic stagnation flows in T-shaped microchannels
}

\author{
J. Soulages ${ }^{a, *}$, M.S.N. Oliveira ${ }^{\text {b }}$, P.C. Sousa ${ }^{\text {b }}$, M.A. Alves ${ }^{b}$, G.H. McKinley ${ }^{a, *}$ \\ a Hatsopoulos Microfluids Laboratory, Department of Mechanical Engineering, Massachusetts Institute of Technology, Cambridge, MA 02139, USA \\ ${ }^{\mathrm{b}}$ Faculdade de Engenharia da Universidade do Porto, Departamento de Engenharia Química, Centro de Estudos de Fenómenos de Transporte, \\ Rua Dr. Roberto Frias, 4200-465 Porto, Portugal
}

\section{A R T I C L E I N F O}

\section{Article history:}

Received 20 March 2009

Received in revised form 5 June 2009

Accepted 8 June 2009

\section{Keywords:}

Microfluidics

Polyethylene oxide (PEO)

Particle image velocimetry

Flow instability

Symmetry-breaking bifurcation

Simplified Phan-Thien-Tanner model

\begin{abstract}
A B S T R A C T
We investigate the stability of steady planar stagnation flows of a dilute polyethylene oxide (PEO) solution using T-shaped microchannels. The precise flow rate control and well-defined geometries achievable with microfluidic fabrication technologies enable us to make detailed observations of the onset of elastically driven flow asymmetries in steady flows with strong planar elongational characteristics. We consider two different stagnation flow geometries; corresponding to T-shaped microchannels with, and without, a recirculating cavity region. In the former case, the stagnation point is located on a free streamline, whereas in the absence of a recirculating cavity the stagnation point at the separating streamline is pinned at the confining wall of the microchannel. The kinematic differences in these two configurations affect the resulting polymeric stress fields and control the critical conditions and spatiotemporal dynamics of the resulting viscoelastic flow instability. In the free stagnation point flow, a strand of highly oriented polymeric material is formed in the region of strong planar extensional flow. This leads to a symmetrybreaking bifurcation at moderate Weissenberg numbers followed by the onset of three-dimensional flow at high Weissenberg numbers, which can be visualized using streak-imaging and microparticle image velocimetry. When the stagnation point is pinned at the wall this symmetry-breaking transition is suppressed and the flow transitions directly to a three-dimensional time-dependent flow at an intermediate flow rate. The spatial characteristics of these purely elastic flow transitions are compared quantitatively to the predictions of two-dimensional viscoelastic numerical simulations using a single-mode simplified Phan-Thien-Tanner (SPTT) model.
\end{abstract}

(c) 2009 Elsevier B.V. All rights reserved.

\section{Introduction}

The continuous miniaturization of flow geometries achievable through microfluidic fabrication techniques has multiple processing advantages including decreased manufacturing costs, fast response times, minimal fluid volumes, precise control over multiphase morphology and increased separation efficiency [1,2]. In particular, microfluidic technology is very relevant to industries associated with genomics, construction of biosensors and lab-ona-chip diagnostics in addition to ink-jet printing [3-5]. Microfluidic technology has also shown its remarkable potential in the field of biochemical analysis and constitutes a valuable tool for separation or mixing, automation and integration of complex chemical and biological assays [6,7]. In these applications, many of the fluids of interest are non-Newtonian in character and understanding

\footnotetext{
* Corresponding authors.

E-mail addresses: soulages@mit.edu (J. Soulages), gareth@mit.edu (G.H. McKinley).
}

their flow behavior at the microscale is important to the design and optimization of the resulting microfluidic devices.

The wide range of deformation rates that can be attained (through precise control of the imposed fluid flow rate and the small characteristic length-scales of the geometry) coupled with the ability to directly image the resulting flow field also make microfluidic devices good platforms for constructing rheometers and flow chambers that enable a systematic investigation of nonNewtonian effects. An overview of several canonical flow types and the challenges associated with quantitative microfluidic rheometry can be found in [8]. In the present work, we focus on two different T-shaped microchannels that are specially constructed to enable an investigation of viscoelastic effects on the stability of planar elongation flows. The channels are fabricated in such a way that the character of the stagnation flow near the separating streamline is modified by the presence, or absence, of a rectangular cavity. The recirculating flow in this cavity is separated from the free stream by a bounding streamline and this changes the local character of the flow near the stagnation point. To illustrate this graphically, we show in Fig. 1(a) and (b) computational predictions of the expected 


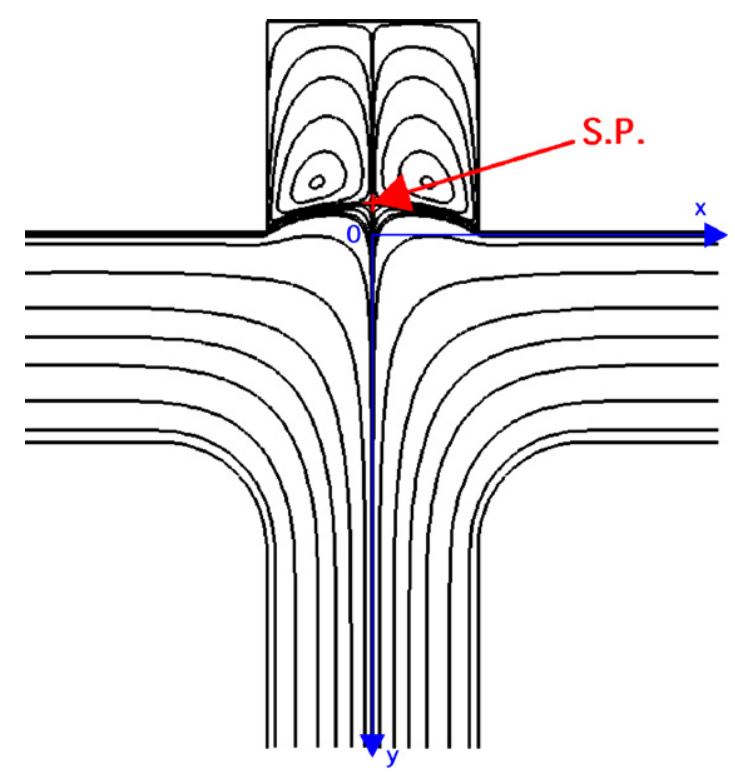

(a) Microchannel with cavity.

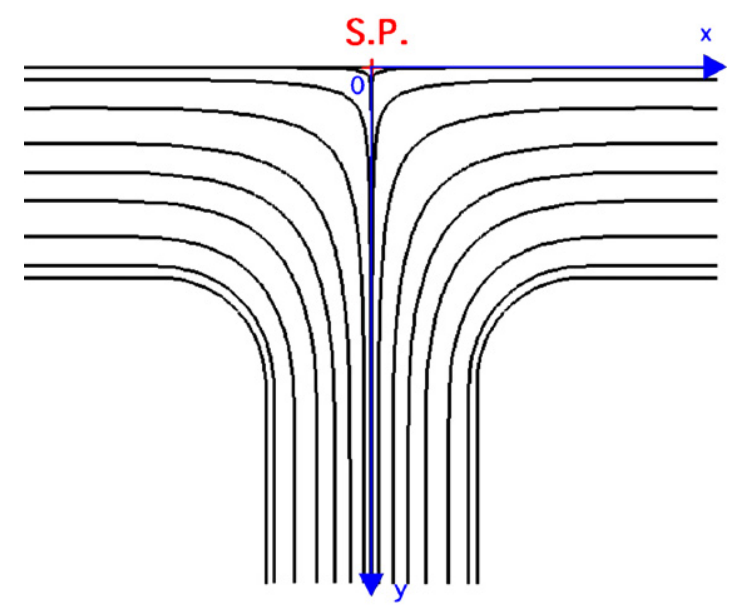

(b) Microchannel without cavity.

Fig. 1. Streamline predictions for Newtonian flow within a T-shaped microchannel with and without a recirculating cavity: $Q=6 \mu \mathrm{L} / \mathrm{h}$; S.P. denotes the location of the stagnation point $\left(R e=2.05 \times 10^{-3}\right)$. (a) Microchannel with cavity. (b) Microchannel without cavity.

streamlines for steady viscous flow of a Newtonian fluid within a T-shaped microchannel with and without a recirculating cavity at $R e=2.05 \times 10^{-3}$. In the absence of the cavity, the stagnation point is located along the symmetry line at the intersection point of the channel sidewall and the separating streamline. As a consequence of the no-slip boundary condition and continuity, the local velocity vector and all velocity gradients are zero at the stagnation point. By contrast, the presence of the cavity and the unconstrained dividing streamline leads to a non-zero velocity gradient at the origin in Fig. 1(a) and the stagnation point is free to move. If we denote the location of the pinned stagnation point as the origin of the laboratory frame (as shown in Fig. 1(b)) then the presence of a free or "unpinned" stagnation point leads to a small vertical displacement towards negative $y$-values inside the cavity.

Both of these flow geometries feature curved streamlines and generate a (nonhomogeneous) planar extensional flow near the stagnation point. These conditions can promote purely elastic flow instabilities $[9,10]$. The T-channel geometry has also been suggested as a suitable geometry for constructing a microfluidic rheometer if the total pressure drop associated with steady symmetric flow of a non-Newtonian fluid is measured [11]. The presence, or absence, of the recirculating cavity thus allows us to focus on the global kinematic consequences that result from fluid viscoelasticity and from local changes in the stagnation flow region. Furthermore, the identical upstream flow conditions in each geometry results in a well-defined pre-shearing history which can be important if a viscoelastic fluid is studied in place of a simple viscous liquid. Similar elongational flows with "pinned" and "free" stagnation points arise in the wakes of objects such as cylinders/spheres and behind rising bubbles $[12,13]$. In the case of a rising bubble, fluid elasticity leads to the formation of a cusp and a symmetry-breaking instability that can be observed experimentally [14] and studied computationally [15]. The unsteady Lagrangian nature of the flow near the rising bubble however complicates the systematic experimental study of the wake near a free stagnation point.

Creeping flows in, and past, cavities have been studied extensively at the macroscale. Pan and Acrivos [16] explored the evolution in the vortex strengths as the cavity depth was changed and Taneda [17] performed an extensive photographic study of the effects of the cavity breadth to height ratio on the formation of vortices inside a cavity using viscous Newtonian liquids such as silicone oil and glycerine. The work of Perera et al. [18] is an example of early numerical work on the effect of elasticity for steady 2D flow in macroscale L-shaped and T-shaped channels. They showed that elasticity only leads to slight deviations in the streamline patterns at low Reynold numbers compared to the Newtonian case. The study of Nishimura and coworkers [19] represents an early combined experimental and numerical study of 2D viscoelastic flow in T-shaped channels using streakline imaging. They studied the effects of elasticity by comparing the flow patterns for a viscoelastic polyacrylamide aqueous solution and a Newtonian dextrose syrup seeded with aluminum powder. They experimentally observed a lip vortex at the re-entrant channel corners in the flows of the polyacrylamide solution. Numerical simulations with the upper-convected Maxwell model at $W i \leq 0.2$ were able to capture qualitatively the viscoelastic distortion in the streamlines, but not the formation of a lip vortex. Binding et al. [20] also investigated viscoelastic creeping flow in a T-junction and past a cavity. They showed that compared to the Newtonian symmetric behavior, the flow of a highly elastic Boger fluid past a cavity clearly became asymmetric beyond a critical flow rate. Using the same flow geometry, they created a stagnation flow by having flow in the two opposing arms of the Tjunction. They observed the formation of lip vortices in the case of the flow of a shear-thinning polymer solution while such vortices were absent for the Boger fluid. In the present study, we aim to characterize the onset of elastic instabilities in similar geometries but at the microscale.

Utilizing microfluidic channels to explore such flows offers the possibility of exploring new regimes of parameter space, that are not readily accessible in macroscale experiments [21]. The relevant dimensionless groups used to characterize a viscoelastic stagnation flow are the Reynolds number $(R e)$, the Weissenberg number $(W i)$ and an elasticity number $(E l=W i / R e)$. An appropriate Reynolds number can be calculated according to $R e=\rho \bar{V} D_{h} / \eta_{0}$, where $D_{h}$ represents the hydraulic diameter of the flow channel and $\rho$ and $\eta_{0}$ represent the fluid density and zero-shear rate viscosity, respectively. Viscoelastic effects in the geometry can be characterized using a Weissenberg number $W i=\lambda \dot{\gamma}$ where $\dot{\gamma}=\bar{V} / \ell$ is an appropriate estimate of the characteristic deformation rate based on the average velocity at the channel inlet, $\bar{V}$, and the relevant lengthscale $\ell$ controlling the kinematics of the stagnation region. The elasticity number $E l=W i / R e=\lambda \eta_{0} / \rho \ell D_{h}$, defined as the ratio of the Weissenberg to Reynolds number, is a measure of the relative importance of elastic to inertial effects, and depends only on the experimental geometry and the material 
properties of the fluid being studied. With the small geometric length scales characteristic of microfluidic geometries it is possible to probe strong elastic effects in the absence of inertial effects; for example in the micro-fabricated planar contractions of Rodd et al. [21,22], elasticity numbers as high as $E l=89$ could be achieved.

Because inertial effects are small, microfluidic devices also provide good platforms to study "purely elastic instabilities" that can arise from the combination of curved streamlines and large tensile viscoelastic stresses $[23,24]$. The time-dependent threedimensional flow that sometimes ensues following onset of a purely elastic flow instability can greatly enhance the mixing efficiency of a microfluidic device at small Reynolds number [25]. There have been few studies to date that have systematically investigated the dynamics associated with these elastic nonlinearities on the microscale [21,26-30]. With microfluidic computing in mind, Groisman et al. were the first to exploit elastic instabilities in designing a nonlinear fluid resistor, a bistable flip-flop memory element [28] and a flow rectifier [29]. Reviews of efforts made to develop nonlinear fluidic logic elements using Newtonian fluids such as water or air can be found in [31,32].

The T-channel design considered in the present work is obviously closely connected to the "cross-slot" configuration which has been used extensively in rheological studies of steady planar elongation flow $[8,33,34]$. In either geometry, the combination of streamline curvature and large extensional deformations near the stagnation point may be anticipated to result in large viscoelastic effects within the flow. The loss of symmetry in a microfluidic crossslot flow at high flow rates is evident in the micellar experiments of Pathak and Hudson [34]. Arratia et al. have documented the existence of a purely elastic instabilities for the case of the cross-slot flow of a polyacrylamide dilute solution [30]. They observed two distinct flow regimes at very small Reynolds numbers $\left(R e \leq 10^{-2}\right)$ : a symmetry-breaking bistable bifurcation for $W i \simeq 4.5$ followed by broadband temporal fluctuations at $W i \gtrsim 12.5$. Very recently these observations of viscoelastic symmetry-breaking have been validated numerically by Poole et al. [35]. By using the upperconvected Maxwell model they demonstrated the purely elastic nature of the flow transition and reported that inertia had a stabilizing effect, delaying the onset of the steady asymmetric flow to higher Wi.

In the present work we seek to compare, quantitatively, experimental observations and numerical computations of this viscoelastic symmetry-breaking transition. By selecting T-channels with, and without, recirculating cavities we can explore the importance of the local planar elongational flow near a "free" and "pinned" stagnation point, respectively. The experiments are performed with a well-characterized dilute aqueous solution of monodisperse PEO and the 2D calculations are performed using a prototypical nonlinear constitutive model with parameters selected to fit the viscometric properties of the test fluid. In Section 2, we describe the fabrication of the test geometries, the imaging techniques and the characterization of the test fluid rheology. In Section 3, we briefly describe the numerical method and then investigate the magnitude of the "birefringent strand" that is generated in the two different planar elongation flows. In Section 4 , we compare streak-imaging measurements and numerical calculations of the streamlines for each microfluidic geometry as the flow rate (and corresponding Weissenberg number) is incremented. In the presence of a recirculating cavity, a symmetry-breaking transition is observed experimentally and predicted computationally at a critical Weissenberg number. By contrast, in the absence of a cavity, the flow near the dividing streamline remains stable and symmetric to substantially higher flow rates before losing stability to three-dimensional and time-dependent perturbations.

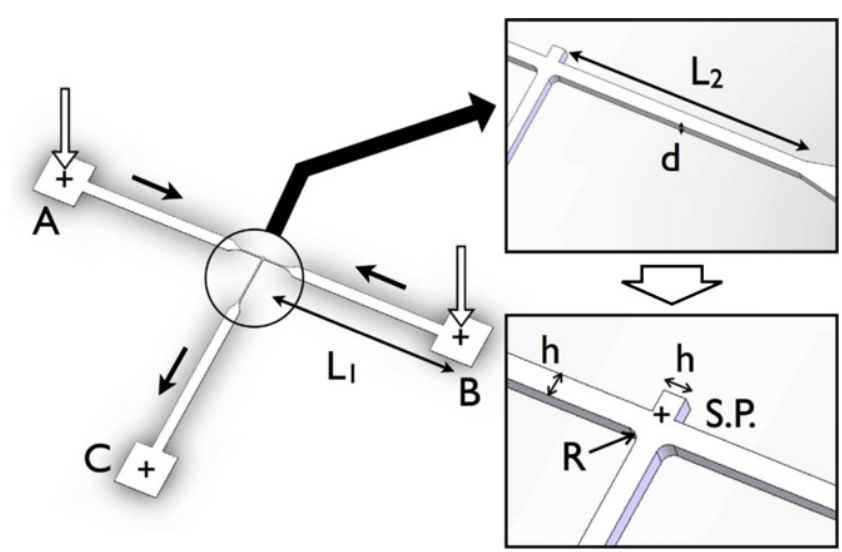

Fig. 2. Schematic diagram of the T-shaped microchannel with a cavity; (A) and (B) are the channel inlets; $(C)$ is the channel outlet; $L_{1}$ the length of the entry channel; $L_{2}$ the entrance length of the T-channel; $d$ the channel depth; $h$ the channel height and width of the square cavity; $R$ the radius of the rounded corners, and S.P. denotes the stagnation point.

\section{Experimental}

In order to perform quantitative comparisons between experimental measurements and numerical computations, it is essential to carefully determine all geometrical and rheological parameters as well as clearly define appropriate dimensionless measures of elasticity and inertia.

\subsection{Microfluidic stagnation flows and dimensionless groups}

The appropriate Reynolds number for this pressure-driven channel flow is calculated according to $\operatorname{Re}=\left(\rho Q D_{h}\right) /\left(h d \eta_{0}\right)$, where $D_{h}$ represents the hydraulic diameter, $D_{h}=2 d h /(d+h), h$ and $d$ are respectively the channel width and depth as shown in Fig. 2. The material properties $\rho$ and $\eta_{0}$ represent the solution density and zero-shear rate viscosity, respectively, and are given in Table 1 . We characterize the elastic effects in the stagnation flow using a Weissenberg number defined as $W i=\lambda_{\text {CaBER }} \dot{\gamma}=\lambda_{\text {CaBER }} \bar{V} /(h / 2)=$ $\left(2 Q \lambda_{\text {CaBER }}\right) / d h^{2}$, where $\lambda_{\text {CaBER }}$ is the relaxation time determined from CaBER measurements (cf. Section 2.3), $\dot{\gamma}$ is the shear rate based on the average velocity at the channel inlets, $\bar{V}=Q / d h$ and a representative length scale for the local stagnation flow suggests $\ell=h / 2$. The elasticity number $E l$, defined as the ratio of the Weissenberg to Reynolds number, is a measure of the relative importance of elastic to inertial effects: $E l=W i / R e$. $E l$ depends only on the experimental geometry and the material properties of the investigated fluid. In our work, the elasticity number $E l=8.61 \times 10^{2}$ is very large so that the elastic stresses dominate compared to inertial effects. Thus, our flow geometries allow us to probe elastically driven flow transitions and instabilities that arise due to the presence of bending streamlines and large tensile viscoelastic stresses in the absence of inertia $[23,24]$.

Table 1

Working fluid rheological properties at $23^{\circ} \mathrm{C}$.

\begin{tabular}{lll}
\hline Zero-shear rate viscosity & $\eta_{0}(\mathrm{mPas})$ & 19.5 \\
Solvent viscosity & $\eta_{S}(\mathrm{mPas})$ & 9.8 \\
Polymer viscosity & $\eta_{P}(\mathrm{mPa} \mathrm{s})$ & 9.7 \\
Zimm relaxation time & $\lambda_{\text {Zimm }}(\mathrm{ms})$ & 2.3 \\
CaBER relaxation time & $\lambda_{\text {CaBER }}(\mathrm{ms})$ & 66 \\
Intrinsic viscosity & {$[\eta](\mathrm{mL} / \mathrm{g})$} & 582 \\
Density & $\rho\left(\mathrm{kg} / \mathrm{m}^{3}\right)$ & 1196 \\
Polymer concentration & $c(\mathrm{~g} / \mathrm{mL})$ & $8.97 \times 10^{-4}$ \\
Concentration ratio & $c / c^{\star}$ & 0.68 \\
\hline
\end{tabular}




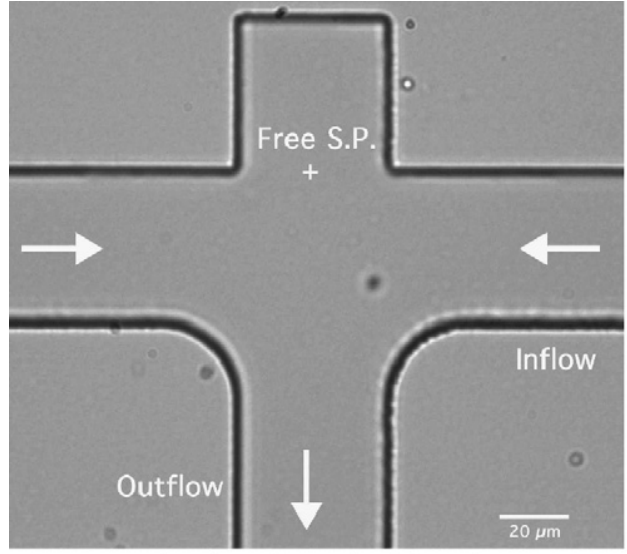

(a) Microchannel with cavity.

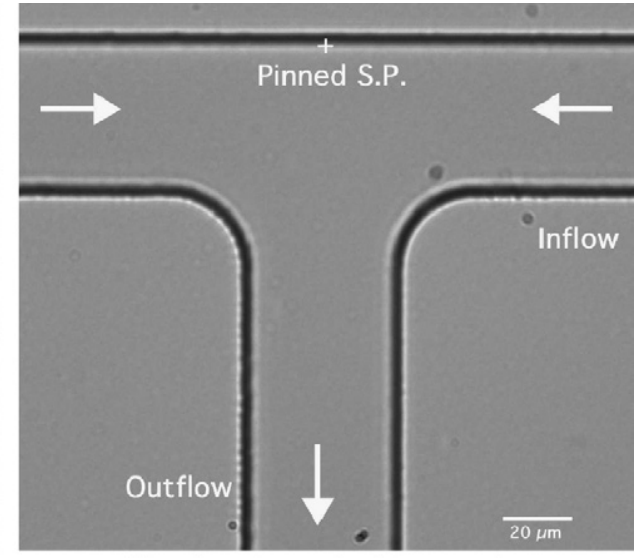

(b) Microchannel without cavity.

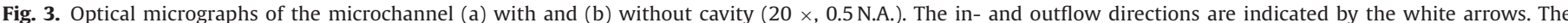

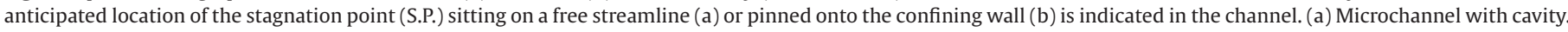
(b) Microchannel without cavity.

\subsection{Microchannel geometry and fabrication}

The relevant variables and dimensions of the micro-fabricated channels used in this study are given in Fig. 2 for the case of the microchannel with a recirculating cavity. The working fluid is injected at the inlets (A) and (B) and exits the channel through the outlet (C). The fluid is directed to the entrances of the central Tjunction circled in the figure by means of two entry channels of length $L_{1}=7 \mathrm{~mm}$. It then enters each side of the T-shaped region and travels a distance $L_{2}=1 \mathrm{~mm}$ before reaching the stagnation point (S.P.) region.

The channel width $h$ and depth $d$ are both equal to $50 \mu \mathrm{m}$. For the entire range of Reynolds numbers investigated in this work, this distance $L_{2}$ is more than 30 times larger than the entrance length $L_{e}$ needed to reach fully developed Newtonian flow, which is given by $L_{e}=D_{h}[0.6 /(1+0.035 R e)+0.056 R e]=30 \mu \mathrm{m}$, where the hydraulic diameter $D_{h}$ coincides with the channel width $h$ for our particular geometry [36]. The square cavity has a length equal to $h$ and the corners of the outflow channel are rounded with a radius $R=25 \mu \mathrm{m}$ in order to guarantee a smooth transition between the inflow and outflow regions. The T-shaped microchannels were fabricated from polydimethylsiloxane (PDMS) using soft-lithography techniques and SU-8 photoresist molds [37-40]. Light micrographs of the microchannels with and without a cavity are shown in Fig. 3(a) and (b), respectively. As discussed in Section 1, the two channel designs differ in the location of the stagnation point: in the presence of the cavity it sits on a "free" streamline whereas it is pinned on the wall of the channel without the recirculating cavity.
A detailed description of the microchannel fabrication procedure is given elsewhere [41]. The use of a contrast enhancement material (CEM388SS, Shin-Etsu MicroSi) allows us to achieve welldefined geometries as shown in Fig. 4(a) with almost perfectly vertical channel sidewalls (the tapering angle is uniformly less than $5^{\circ}$ as illustrated in Fig. 4(b)).

\subsection{Test fluid rheological characterization}

The test fluid used in the present experiments is a dilute polymer solution of a high molecular weight polyethylene oxide (0.075 wt.\%) with a relatively narrow molecular weight distribution (PEO, $M_{w}=2 \times 10^{6} \mathrm{~g} / \mathrm{mol}$, polydispersity index $M_{w} / M_{n}=1.13$ [22], Aldrich) in a glycerol/water mixture (60/40 wt.\%). The rheological properties of the PEO solution were characterized in both steady shear and transient uniaxial extension. The polymeric solution and solvent zero-shear rate viscosities were obtained from viscometric experiments in a double gap Couette geometry using a controlled stress rheometer (AR-G2, TA Instruments). The steady shear data were measured at $23^{\circ} \mathrm{C}$ for shear rates in the range $1 \leq \dot{\gamma} \leq 10,000 \mathrm{~s}^{-1}$ and are presented in Fig. 5 . The PEO solution has a zero-shear rate viscosity $\eta_{0}=19.5 \mathrm{mPa}$ s and is weakly shear thinning for shear rates $\dot{\gamma} \geq 15 \mathrm{~s}^{-1}$. This gives a coarse estimate of a characteristic relaxation time $\lambda \simeq 1 / 15 \mathrm{~s}^{-1}=67 \mathrm{~ms}$ which is in good agreement with the relaxation time determined from CaBER measurements in Fig. 6. The zero-shear rate viscosity of the solvent is $\eta_{S}=9.8 \mathrm{mPa} s$ resulting in a total polymeric contribution to the zero-shear rate viscosity of $\eta_{P}=9.7 \mathrm{mPa}$. The predictions
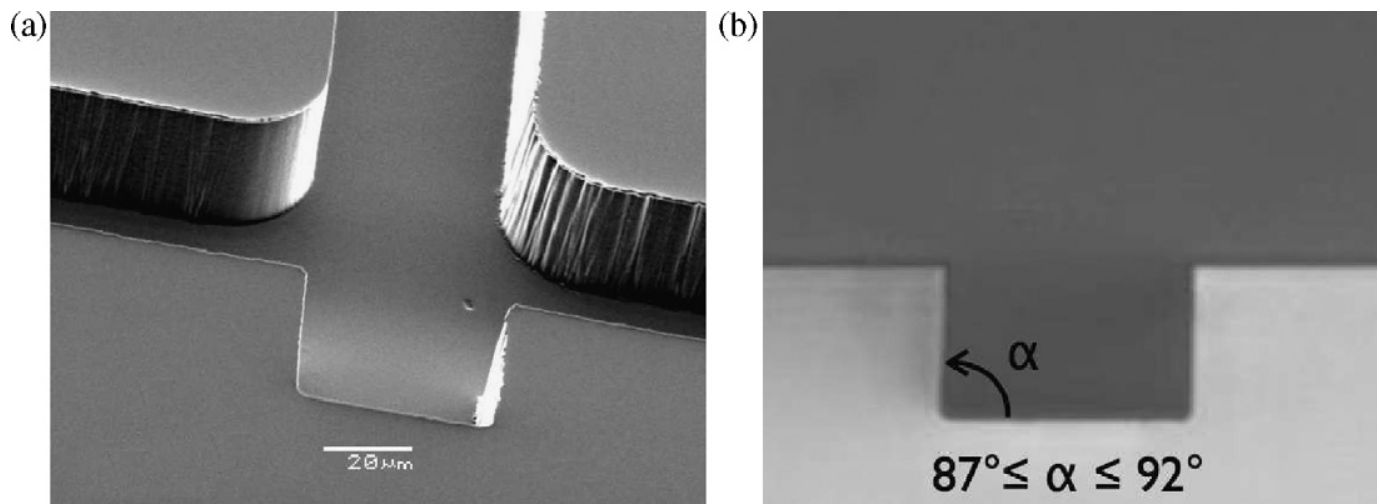

Fig. 4. Microchannel SEM image (a) and optical micrograph of the channel cross-section (b) showing the well-defined geometries achievable with soft lithography. 


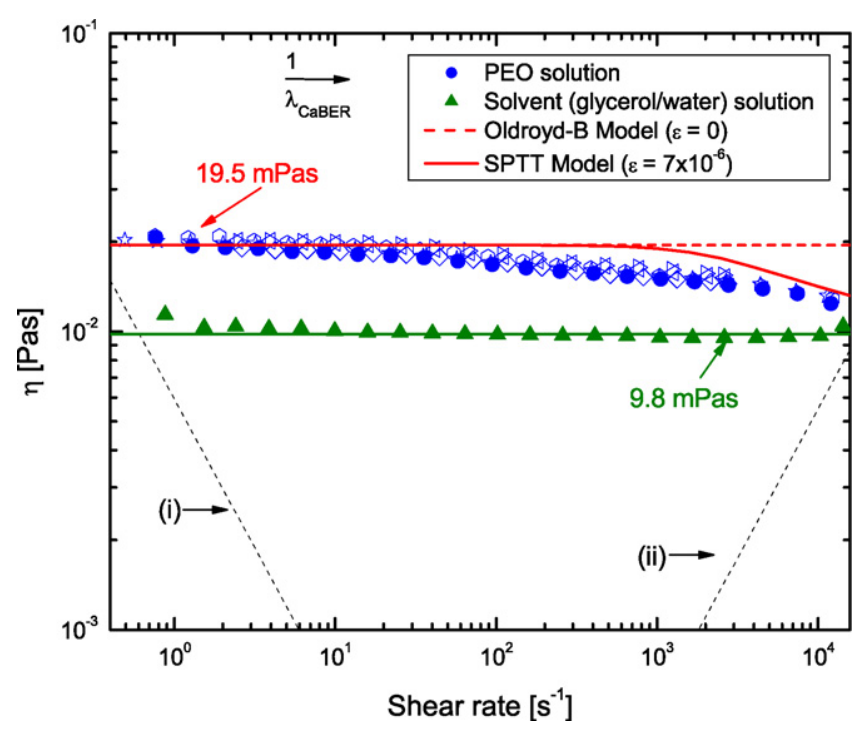

Fig. 5. Steady shear data measured at $23^{\circ} \mathrm{C}$ in a double gap Couette geometry using a controlled stress rheometer (AR-G2, TA Instruments). In the case of the PEO solution, the open symbols represent repeated experiments. The SPTT model predictions are shown by the red dashed and solid lines for $\varepsilon=0$ (Oldroyd-B model) and $\varepsilon=7.0 \times 10^{-6}$, respectively. (i) Minimum measurable shear viscosity based on 20 times the minimum torque resolvable by the rheometer $\left(2 \times 10^{-6} \mathrm{~N} \mathrm{~m}\right)$; (ii) maximum measurable shear viscosity before the onset of Taylor instabilities; $\lambda_{\text {CaBER }}$ : relaxation time determined from CaBER measurements as shown in Fig. 6.

of the SPTT model are shown by the red dashed and solid lines, respectively, for $\varepsilon=0$ (Oldroyd-B model) and $\varepsilon=7.0 \times 10^{-6}$.

Also represented in Fig. 5 are the lower and upper limits of the shear data based on the rheometer torque transducer specifications and the onset of Taylor instabilities as described in [21]. According to a linear stability analysis [42], the critical Taylor number at the onset of inertial instabilities for a Newtonian fluid in the Couette geometry is given by $T a_{\text {crit }} \simeq 2 R e^{2} \phi=3400$, where Re denotes the Reynolds number and $\phi=d / R_{\mathrm{in}}$ is the ratio of the gap width

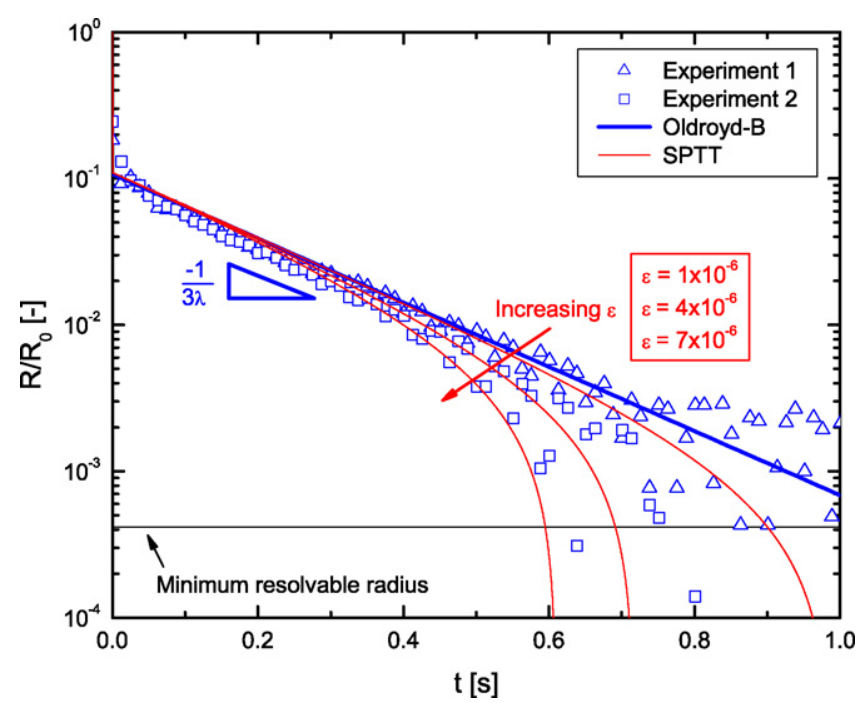

Fig. 6. Time evolution of the mid-point diameter of the fluid thread in a CaBER experiment at $23^{\circ} \mathrm{C}$ (open symbols). The blue (thick) solid line represents the exponential fit to the experimental data (Oldroyd-B model) from which the longest relaxation time is extracted: $\lambda=66 \pm 4 \mathrm{~ms}$. The horizontal black solid line indicates the minimum resolvable radius ratio based on the CaBER laser micrometer resolution of $2.5 \mu \mathrm{m}$ (Omron Z4LA). Predictions of the SPTT model for different values of the extensibility parameter $\varepsilon$ are also shown in the figure (red solid lines). (For interpretation of the references to colour in this figure legend, the reader is referred to the web version of the article.) $d$ and the radius of the inner cylinder $R_{\mathrm{in}}$. The Reynolds number for circular Couette flow is defined as $\operatorname{Re}=\rho \Omega_{\text {in }} R_{\text {in }} d / \eta(\dot{\gamma})$, where $\Omega_{\text {in }}$ represents the angular velocity of the inner cylinder, $\rho$ is the density and $\eta(\dot{\gamma})$ is the (shear-rate-dependent) viscosity of the PEO solution. For $R_{\mathrm{in}}=22 \mathrm{~mm}$ (outer radius of the rotor), $d=0.38 \mathrm{~mm}$, $\rho=1196 \mathrm{~kg} / \mathrm{m}^{3}$, the criterion for the onset of Taylor instabilities can be rewritten as $\eta(\dot{\gamma})=5.5 \times 10^{-7} \dot{\gamma}$, where $\eta$ is in Pa s and $\dot{\gamma}$ in $\mathrm{s}^{-1}$. This equation is represented by the dashed line labeled (ii) in Fig. 5 .

The characteristic relaxation time of the solution was determined from capillary breakup extensional rheometry (CaBER) measurements as illustrated in Fig. 6. A thorough description of this technique can be found in [43-45]. Following the nomenclature of [45], the CaBER geometrical configuration used in the present study was such that the initial height was $h_{0}=2.11 \mathrm{~mm}$ $\left(\Lambda_{0}=h_{0} / 2 R_{0}=0.35\right)$ and the final aspect ratio was $\Lambda_{f}=1.57$, corresponding to an imposed step strain of $\epsilon=\ln \left(\Lambda_{f} / \Lambda_{0}\right)=1.50$. In Fig. 6 , the blue (thick) solid line represents the fit to the measured evolution of the filament diameter using a single exponential decay and based on the Oldroyd-B model [44]. The resulting relaxation time equals $\lambda_{\text {CaBER }}=66 \pm 4 \mathrm{~ms}$.

Also shown in Fig. 6 are the results of the 1D calculations with the SPTT model. This model (see Section 3.1 for details) contains a single nonlinear constitutive parameter $(\varepsilon)$ which controls the magnitude of strain-hardening in the extensional viscosity of the fluid $\left(\eta_{E} \simeq 2 \eta_{P} / \varepsilon\right.$ for small $\varepsilon$ [46]). As the polymer chains in the thinning thread approach full extension, the filament radius no longer thins exponentially; but instead decreases linearly in time [47]. This deviation from exponential behavior allows us to determine a bound on the range of values of $\varepsilon$ characterizing the $0.075 \mathrm{wt}$.\% PEO solution. From the data and simulations shown in Fig. 6 , it is clear that the PEO molecules are highly extensible with $0 \leq \varepsilon \leq 7.0 \times 10^{-6}$. Any further increase in $\varepsilon$ restricts the region of exponential decay and reduces the predicted time to breakup to unphysically small values.

As observed in $[21,22,47]$ and due to "self-concentration" effects, the relaxation time for polymer solutions determined in CaBER measurements is significantly greater than the relaxation time determined according to the Zimm theory, which is expressed by [48]:

$\lambda_{\text {Zimm }}=F \frac{[\eta] M_{w} \eta_{S}}{N_{A} k_{B} T}$,

where $M_{w}$ is the polymer molecular weight, $N_{A}$ is Avogadro's number, $k_{B}$ the Boltzmann constant, $T$ the absolute temperature, and $[\eta]$ is the intrinsic viscosity determined from U-tube capillary viscosimeter experiments in [22]. The prefactor $F$ can be approximated by the Riemann zeta function $F=\zeta^{-1}(3 v)=1 / \Sigma_{i=1}^{\infty}\left(1 / i^{3 v}\right)$, in which $v$ represents the solvent quality exponent and is $v \simeq 0.55$ for PEO in glycerol/water so that $F \simeq 0.46$ [48].

All of the fluid rheological properties are summarized in Table 1. The density was determined using calibrated $5 \mathrm{~mL}$ density flasks in [22]. The overlap concentration $c^{\star}$ was calculated according to the expression $c^{\star}=0.77 /[\eta]$ (see Graessley [49] for discussion) and is equal to $c^{\star} \simeq 1300 \mathrm{ppm}$. On this basis, the $0.075 \mathrm{wt}$.\% PEO solution can thus be considered as dilute $\left(c / c^{\star}=0.68\right)$. From independent measurements of the shear rheology, we also find $\left(\eta_{0}-\eta_{S}\right) / \eta_{S}=$ $9.7 / 9.8 \leq 1$.

\subsection{Flow visualization}

The microparticle image velocimetry experimental setup consists of a CCD camera (mvBlueFOX-120a, Matrix Vision GmbH), an inverted microscope (Nikon, Eclipse TE 2000-S) equipped with a G-2A filter cube (exciter, 535-550 nm; dichroic, $565 \mathrm{~nm}$; long-pass emitter, $590 \mathrm{~nm}$ ) and an external continuous light source (mercury lamp, illumination wavelength: $532 \mathrm{~nm}$ ). The solution is fed to the channel inlets using Tygon tubing by means of two twin syringe 
pumps (New Era Pump Systems, Inc.) and two Hamilton gastight syringes $(500 \mu \mathrm{L}$, diameter: $3.26 \mathrm{~mm}$ ). The PEO solution is seeded with $1.1 \mu \mathrm{m}$ diameter fluorescent tracer particles (Nile Red, Molecular Probes, Invitrogen; Ex/Em: 520/580 nm; $c_{P}=0.02$ wt.\%), which are illuminated by the light source and imaged through the microscope objective $(20 \times, 0.5$ N.A.) onto the CCD array of the camera at a frame rate of $3.81 \mathrm{fps}$ and exposure time of about $250 \mathrm{~ms}$.

Sodium dodecyl sulfate (SDS) from Sigma-Aldrich was added to the PEO solution at a concentration of $c_{\mathrm{SDS}}=0.1 \mathrm{wt}$.\% in order to inhibit the fluorescent tracers from sticking onto the polydimethylsiloxane microchannel walls. The addition of SDS was shown to have a negligible influence on the value of the relaxation time $\lambda_{\text {CaBER }}$ measured from CaBER experiments as well as on the values of both $\eta_{0}$ and $\eta_{s}$.

All of the streakline images presented in this work were recorded at the mid-plane of the microchannel. The physical location of the mid-plane was determined experimentally by successively focusing the image of a fluorescent tracer adhered to the top and bottom surfaces of the channel. The depth over which the tracers contribute to the recorded streamlines is actually given by the measurement depth $\delta z_{m}[21,22,50]$ given by

$\delta z_{m}=\frac{3 n \lambda_{0}}{(\mathrm{NA})^{2}}+2.16 \frac{d_{\mathrm{P}}}{\tan (\theta)}+d_{\mathrm{P}}$.

In Eq. (2), $\lambda_{0}$ represents the wavelength of the emitted light $\left(\lambda_{0}=\right.$ $580 \mathrm{~nm}$ ), $n$ is the medium refractive index (water $n=1.33$ ), NA is the numerical aperture of the objective lens, $d_{\mathrm{p}}$ is the tracer diameter and $\theta$ is defined as $\theta=\sin ^{-1}(\mathrm{NA} / n)$. Eq. (2) is only valid for $d_{\mathrm{P}}>e / \mathrm{M}$, where $e$ and $\mathrm{M}$ respectively denote the minimum resolvable feature size (or the CCD camera pixel size: $7.4 \mu \mathrm{m}$ ) and the objective magnification. In our work, $e / \mathrm{M}=0.37 \mu \mathrm{m}$, which is very small compared to the diameter of the tracer particles $(1.1 \mu \mathrm{m})$. The depth of measurement can thus be determined from Eq. (2) and is $\delta z_{m}=14.5 \mu \mathrm{m}$, which corresponds to approximately $29 \%$ of the total channel depth.

\section{Numerical method and computational meshes}

\subsection{Governing equations and numerical method}

In addition to the experimental measurements, we perform 2Dcalculations to simulate the isothermal flow of the viscoelastic fluid through T-shaped microchannels with and without the recirculating cavity. We use a fully implicit finite-volume method with a time-marching pressure-correction algorithm [51,52] to solve the equations of conservation of mass and momentum:

$\nabla \cdot \mathbf{u}=0$

$\rho\left[\frac{\partial \mathbf{u}}{\partial t}+\mathbf{u} \cdot \nabla \mathbf{u}\right]=-\nabla p+\eta_{\mathrm{S}} \nabla \cdot\left[\nabla \mathbf{u}+(\nabla \mathbf{u})^{\mathrm{T}}\right]+\nabla \cdot \boldsymbol{\tau}$,

together with an appropriate constitutive equation for the polymeric component of the extra-stress, $\boldsymbol{\tau}$. The numerical code used here has been applied extensively in 2D calculations [53,54] and with axisymmetric geometries [55]. Additionally, it has also been used for full three-dimensional (3D) simulations including those of planar channels in which the depth of the channels is kept constant as is typical of microfluidic fabrication $[50,56]$.

Regarding the boundary conditions, we imposed fully developed velocity and stress profiles at the inlets, Neumann boundary conditions at the outlet, and no-slip conditions at the walls. Details of the implementation of boundary conditions can be found in Oliveira et al. [51]. For the discretization of the equations, we use central differences for the diffusive terms and the CUBISTA high-resolution scheme [57] for the convective terms.
In order to simulate a viscoelastic fluid with rheological characteristics matching those of the experimental fluid, we use the linear form of the simplified Phan-Thien-Tanner model (SPTT), for which the polymeric component of the extra-stress tensor is given by Eq. (5):

$$
\left[1+\frac{\lambda \varepsilon}{\eta_{P}} \operatorname{tr}(\boldsymbol{\tau})\right] \boldsymbol{\tau}+\lambda \stackrel{\nabla}{\boldsymbol{\tau}}=2 \eta_{P} \mathbf{D},
$$

where $\boldsymbol{\tau}$ represents the extra-stress tensor and $\operatorname{tr}(\boldsymbol{\tau})$ represents the trace of $\tau$, the symbol () denotes the upper-convected time derivative, $\eta_{P}$ is the polymer contribution to the zero-shear rate viscosity, $\varepsilon$ is the extensibility parameter and $\mathbf{D}$ is the rate-of-deformation tensor. The SPTT model exhibits shear-thinning behavior, elastic normal stresses and a bounded elongational viscosity, and has been shown to be appropriate for modeling both polymeric solutions and polymer melts [58]. In addition, we also perform a number of calculations using the Oldroyd-B model, which is a limiting case of the SPTT model that can be recovered when $\varepsilon=0$.

To enhance numerical stability, we employ the log-conformation tensor approach [59], as described in detail in Afonso et al. [60].

\subsection{Computational meshes and problem definition}

The geometries for the numerical calculations represent $\mathrm{T}$ channels with and without a cavity and are similar to those used experimentally (cf. Section 2.2). The meshes used to map the two domains are block-structured and non-uniform, with the size of each cell relating to its neighbors by a geometric progression within each direction. A zoomed view of the computational meshes near the central region is shown in Fig. 7. The total number of cells $(N C)$ was adjusted according to the configuration used: $N C=12801$ and $N C=10251$ for the T-channels with and without cavity, respectively. Additionally, the smallest cell size was set $\Delta x_{\min }=\Delta y_{\min }=$ $0.02 \mathrm{~h}$ for both configurations.

In the numerical calculations, the characteristics of the fluid were fixed in accordance with the properties of the experimental test fluid presented in Table 1 . The density was fixed at $1196 \mathrm{~kg} / \mathrm{m}^{3}$, the solvent viscosity ratio $\beta=\eta_{S} / \eta_{0}$ was kept constant $(\beta=0.50)$ to match the shear rheometry data; the relaxation time was taken as $\lambda_{\text {CaBER }}=66 \mathrm{~ms}$ as measured in CaBER experiments; while the extensibility parameter of the PTT model was varied between $\varepsilon=0$ and $\varepsilon=7.0 \times 10^{-6}$, according to the fits to the CaBER experimental data shown in Fig. 6. As we demonstrate in Section 4.1 below, the difference between the calculation with $\varepsilon=0$ and $\varepsilon=7.0 \times 10^{-6}$ is negligible for the range of Weissenberg numbers explored. Thus, the uncertainty in the exact estimation of this parameter from CaBER experiments is not critical in the present computations.

\section{Results and discussion}

We first examine the influence of the extensibility parameter $\varepsilon$ on the streamlines predicted by the SPTT model and subsequently analyze the stress field obtained from 2D numerical simulations. We then compare the flow patterns obtained in the T-shaped microchannels with and without a recirculating cavity for both the viscoelastic PEO solution and its glycerol/water solvent Newtonian counterpart. Finally, we characterize the nature of the symmetrybreaking bifurcation observed after a critical Weissenberg number in the T-shaped microchannel containing a recirculating cavity.

\subsection{Extensibility parameter $\varepsilon$}

The effect of the extensibility parameter $\varepsilon$ in Eq. (5) on the predictions of the SPTT model was investigated for two different volumetric flow rates as shown in Fig. 8(a) and (b). For $\varepsilon=0$, the 
Oldroyd-B model is recovered and the resulting streamlines appear as the black dashed lines in Fig. 8(a) and (b). Also plotted as red solid lines in the figures are the streamlines corresponding to the value $\varepsilon=7.0 \times 10^{-6}$ that best captures the time evolution of the midpoint diameter of the PEO solution thread in CaBER experiments as illustrated in Fig. 6. As shown in both figures, the extensibility parameter $\varepsilon$ has little effect on the SPTT model predictions and the streamline patterns corresponding to the two different choices of $\varepsilon$ superpose for both geometries.

From the CaBER experiments presented in Fig. 6, we could determine the range of the extensibility parameters for which a good agreement between the experimental measurements of capillary thinning and the predictions of a single mode constitutive model could be obtained. As the model predictions are not significantly affected by the choice of $\varepsilon$ in that particular range, we use the maximum value of $\varepsilon=7.0 \times 10^{-6}$ for all numerical simulations presented in this work. Indeed, this value is shown to cover the entire range of experimental data from CaBER measurements in Fig. 6.

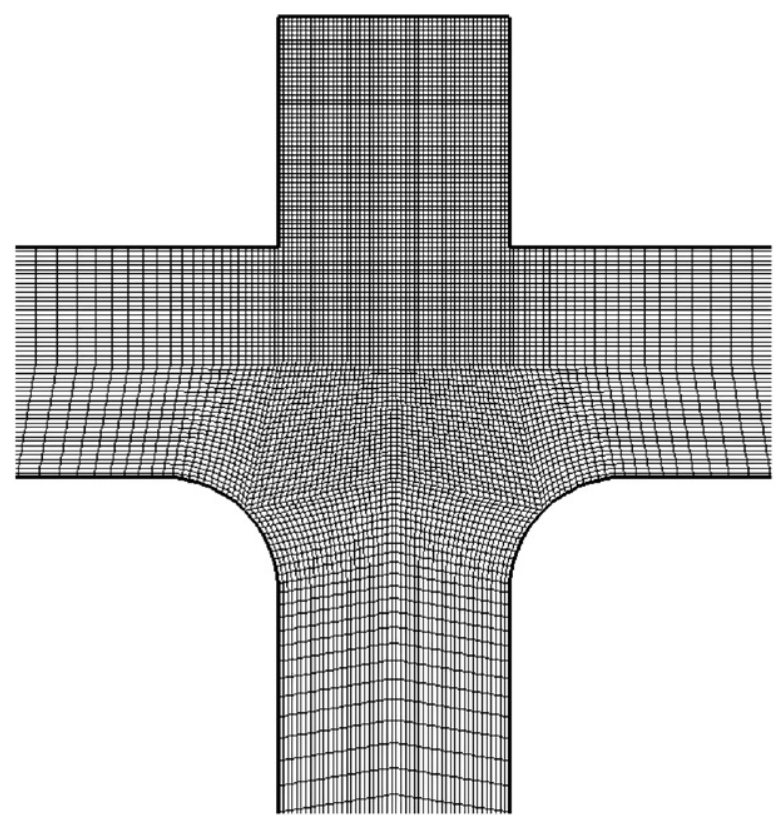

(a) Mesh with recirculating cavity.

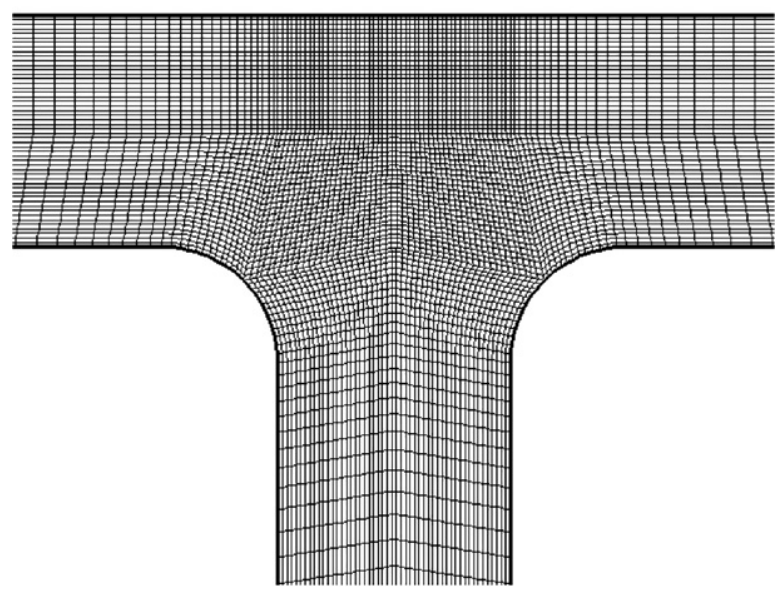

(b) Mesh without recirculating cavity.

Fig. 7. Zoomed view of the computational meshes near the central region for the T-shaped microchannels (a) with, or (b) without recirculating cavity. (a) Mesh with recirculating cavity. (b) Mesh without recirculating cavity.

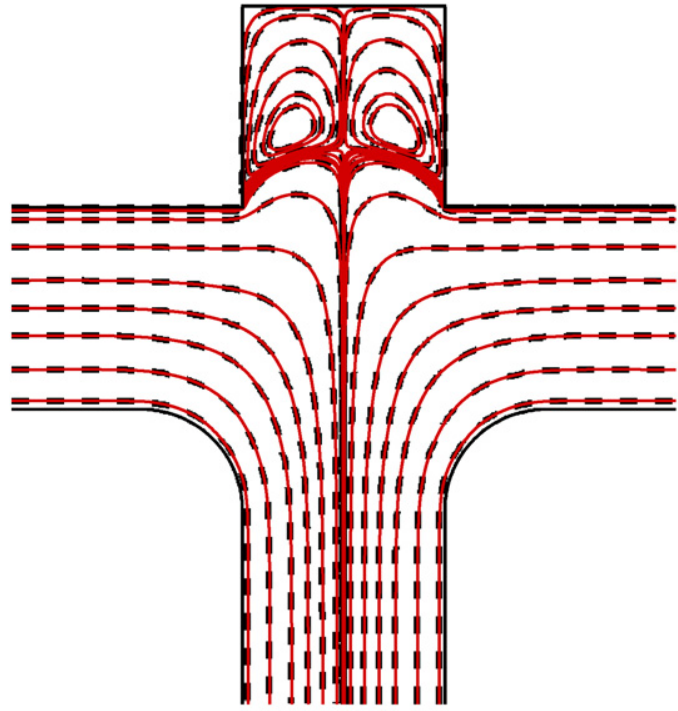

(a) $Q=2.5 \mu \mathrm{L} / \mathrm{hr}$ (cavity).

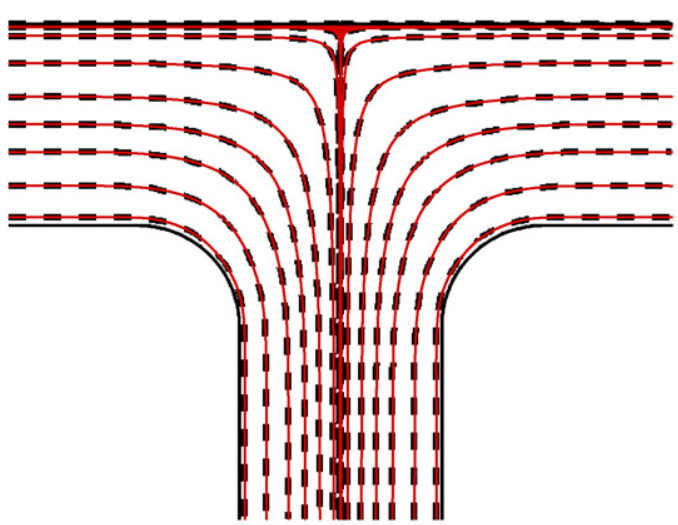

(b) $Q=2.5 \mu \mathrm{L} / \mathrm{hr}$ (no cavity).

Fig. 8. Effect of the SPTT model extensibility parameter $\varepsilon$ on the streamline patterns for the T-shaped microchannel with (a) and without (b) recirculating cavity. The thin red streamlines were obtained with $\varepsilon=7.0 \times 10^{-6}$ and the thick dashed black streamlines correspond to $\varepsilon=0$ (Oldroyd-B model); $R e=8.53 \times 10^{-4}, W i=0.73$. (a) $Q=2.5 \mu \mathrm{L} / \mathrm{h}$ (cavity). (b) $Q=2.5 \mu \mathrm{L} / \mathrm{h}$ (no cavity). (For interpretation of the references to colour in this figure legend, the reader is referred to the web version of the article.)

\subsection{Stress field}

The contour plots of the normalized first normal stress difference for the T-shaped microchannel with and without a recirculating cavity are shown in Fig. 9(a) and (b), respectively. Although not shown in the figures, the exit channel length used in the simulations is $550 \mu \mathrm{m}$ in order to guarantee that the stress field is fully developed in the outlet arm. The normal stress difference is scaled with the characteristic viscous stress $\eta_{0} \bar{V} /(h / 2)$ and is plotted at a fixed Weissenberg number $W i=0.73$

At this Weissenberg number, the numerical solution is symmetric for both geometries, which is in agreement with the symmetry of the streamline images captured under these flow conditions (see Sections 4.3.1 and 4.4.1). A local inhomogeneous planar extensional flow develops where the two streams meet $(x=0)$, which results in a localized birefringent strand of highly stretched material [61]. This strand of oriented material leads to the large normal stress difference observed along the channel centerline. The presence of a recirculating flow in the cavity strongly affects the local kinematics near the stagnation point and leads to a significantly lower tensile 
stress difference along the centerline compared to the pinned stagnation point flow as shown in Fig. 10. When the stagnation point is pinned at the no-slip wall, the dimensionless normal stress difference is $h\left(\tau_{y y}-\tau_{x x}\right) /\left(2 \eta_{0} \bar{V}\right) \geq 70$ whereas it remains under 20 for the case with a recirculating cavity. As shown in the figure, the extensibility parameter $\varepsilon$ has little effect on the numerical predictions of the first normal stress difference for this Weissenberg number. This is because of the limited residence time and moderate total Hencky strains experienced by most material elements; the polymer molecules thus do not approach the finite extensibility limit. In the pinned stagnation point flow, the large stress gradients observed along the channel centerline are very similar to those encountered in the downstream wake of the flow past a confined cylinder in a channel [62-64]. As will be discussed in the following section, these stresses control the onset of the viscoelas-

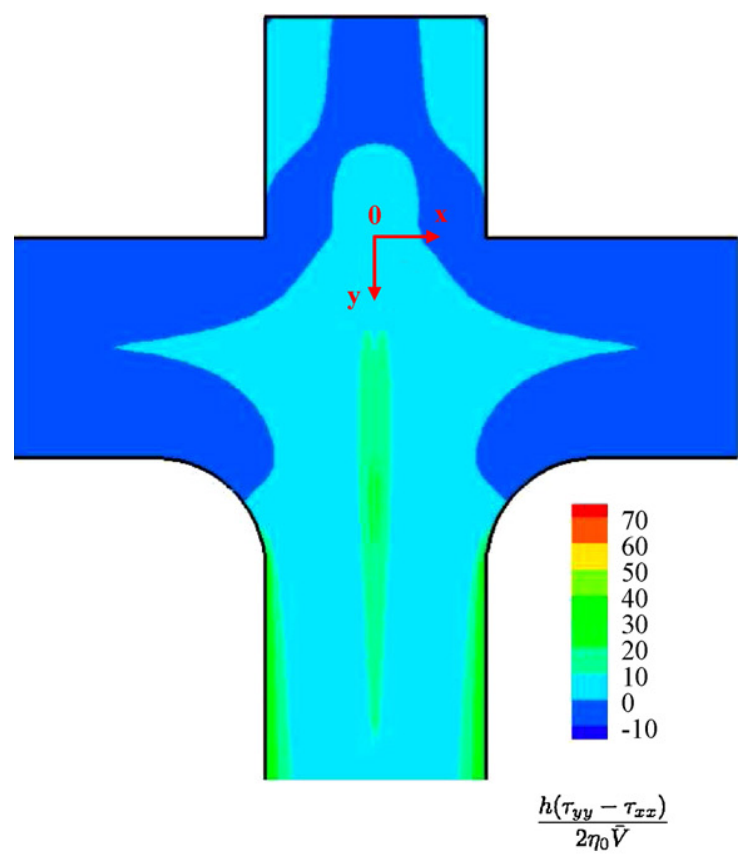

(a) $Q=2.5 \mu \mathrm{L} / \mathrm{hr}$ (cavity).

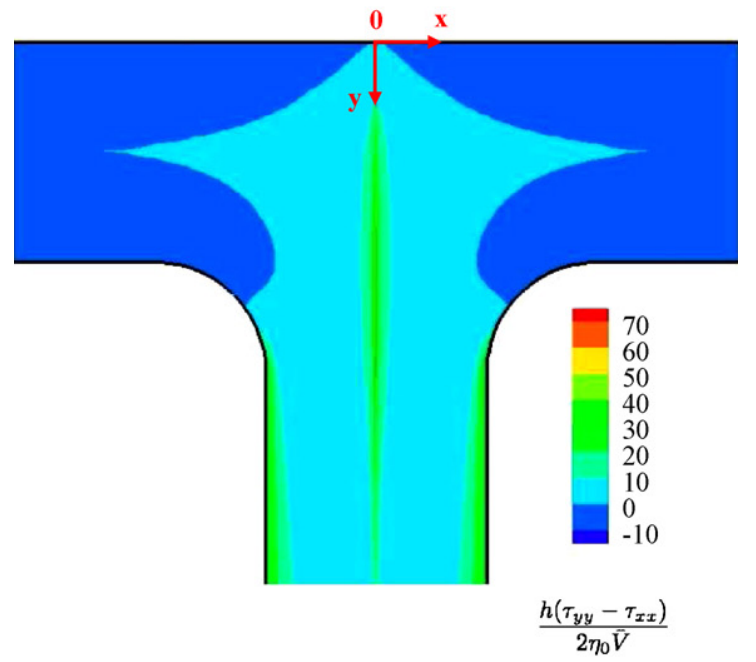

(b) $Q=2.5 \mu \mathrm{L} / \mathrm{hr}$ (no cavity).

Fig. 9. Contour plots of the normalized first normal stress difference ( $h\left(\tau_{y y}-\right.$ $\left.\left.\tau_{x x}\right) /\left(2 \eta_{0} \bar{V}\right)\right)$ for the T-shaped microchannel without (a), and with (b), recirculating cavity (SPTT model; $\beta=0.50, \varepsilon=7.0 \times 10^{-6}, R e=8.53 \times 10^{-4}, W i=0.73$ ). (a) $Q=2.5 \mu \mathrm{L} / \mathrm{h}$ (cavity). (b) $Q=2.5 \mu \mathrm{L} / \mathrm{h}$ (no cavity).

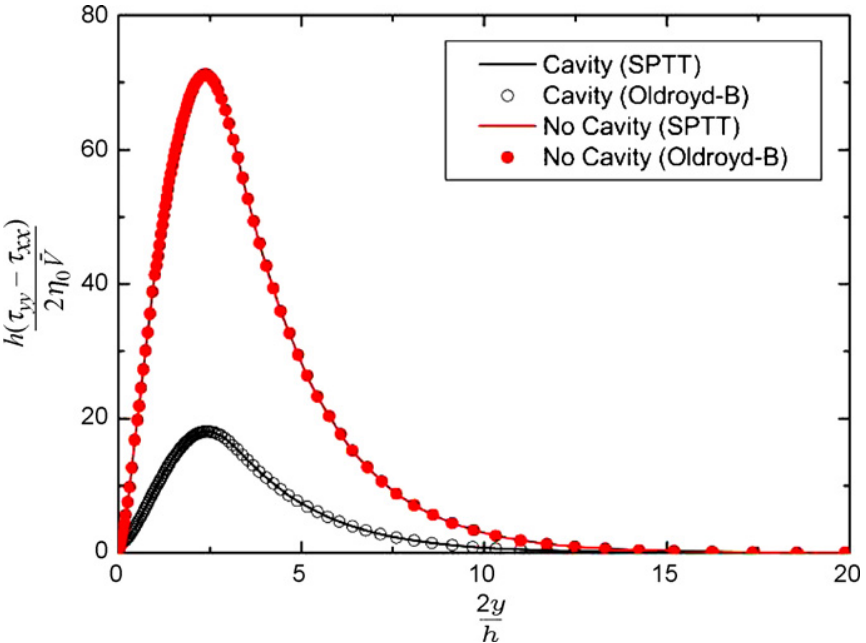

Fig. 10. Line plots of normalized first normal stress difference along the symmetry line $x=0$ from Fig. 9(a) and (b). The solid lines correspond to the SPTT model with $\varepsilon=7.0 \times 10^{-6}$ and the open and filled symbols obtained for $\varepsilon=0$ (Oldroyd-B model) correspond to the microchannel with and without cavity, respectively (for both models: $\beta=0.50, \operatorname{Re}=8.53 \times 10^{-4}, W i=0.73$ ).

tic flow transitions observed experimentally in the two different geometries.

\subsection{Pinned stagnation point flow}

\subsubsection{Viscoelastic and Newtonian flow comparison}

In Fig. 11, we show a comparison of the streamline images obtained at different volumetric flow rates (or equivalently, different Weissenberg numbers in case of the viscoelastic PEO solution) for the aqueous solution of $\mathrm{PEO} /$ glycerol/water and for the corresponding Newtonian solvent. The flow patterns for the Newtonian fluid and the viscoelastic fluid response at a constant elasticity number $E l=W i / R e=861$ are shown in Fig. 11(f)-(j) and (a)-(e), respectively. The aim of this comparison is to demonstrate the effect of elasticity on the stagnation flow where the stagnation point is pinned onto the microchannel confining wall.

As can be seen in Fig. 11(a)-(e), we observe a transition from a symmetric Newtonian-like behavior to an unsteady 3D flow for the viscoelastic PEO solution after a critical Weissenberg number $W i_{\text {crit }} \simeq 3.2$. This unstable $3 \mathrm{D}$ flow is characterized by overlapping streaklines within the measurement depth $\delta z_{m}=14.5 \mu \mathrm{m}$ as shown in Fig. 11(c)-(e). At higher flow rates (Fig. 11(e)), the flow eventually becomes chaotic and is suitable for mixing purposes [65].

The Newtonian flow counterpart remains symmetric and stable for the entire range of volumetric flow rates tested in this work $\left(R e \leq 6.5 \times 10^{-2}\right)$. The stability of the symmetric flow is further visually confirmed by the presence of a non-moving fluorescent tracer particle at the location of the stagnation point in Fig. 11(f)-(j), which is not flushed by the inflow over the course of the experiment, contrary to the other tracer potentially stuck to the wall on the left hand-side of the stagnation point that is only visible in Fig. 11(f)-(h). In the outflow channel, some fluorescent particles stuck onto the surface of the PDMS channel are clearly visible. Even if they do not perturb the symmetry of the flow profile at the channel mid-plane, they further motivate the use of SDS which helps to limit their accumulation at the channel edges and in the recirculating cavity during the streakline imaging experiments.

Comparing the streamline patterns corresponding to the PEO solution and the viscous Newtonian counterpart at low Reynolds numbers, it can be concluded that the transition from a stable $2 \mathrm{D}$ flow to an unsteady $3 \mathrm{D}$ flow is elastically driven and is due 


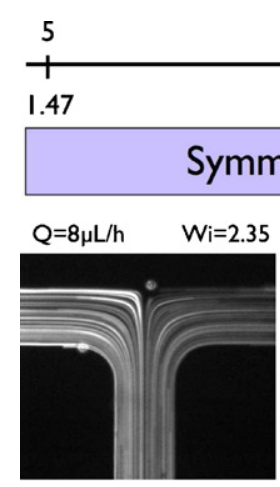

(a)

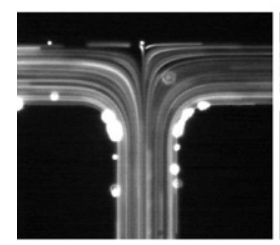

(f)
11

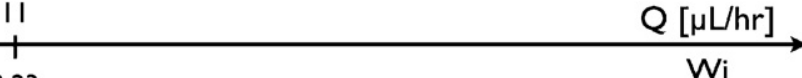

3.23

Unsteady 3D

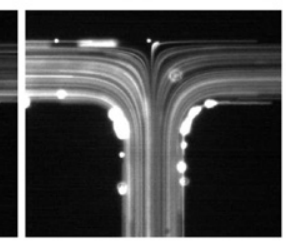

(g)

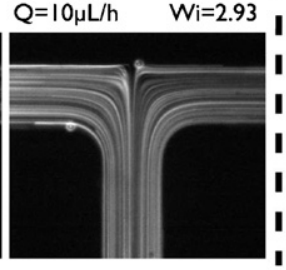

(b)

(c)

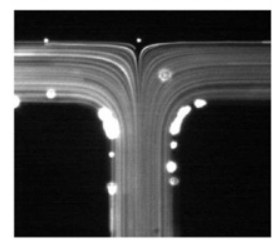

(h)

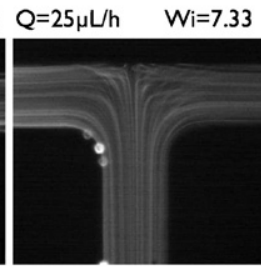

(d)

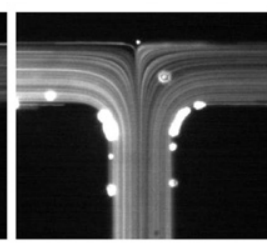

(i)

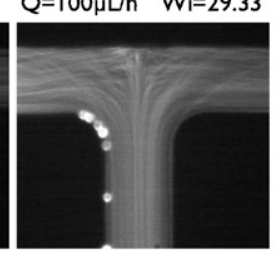

(e)

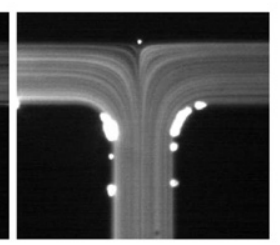

(j)

Fig. 11. Viscoelastic ((a)-(e)) and Newtonian ((f)-(j)) flow streamline patterns as a function of the volumetric flow rate $Q$.

to large stress gradients that develop downstream of the stagnation point as shown in Fig. 9(b). As discussed by Becherer et al. [66], the local planar extensional flow in this region is similar to the wake behind a cylinder confined in a channel for which a number of studies (e.g. [62-64]) report the onset of unsteady flow at $W i \simeq 1$. In the present experiments, we find that when the Weissenberg number exceeds $W i_{\text {crit }} \simeq 3.2$, the flow becomes clearly time-dependent. Movies of this unstable flow regime were also recorded using the mvBlueFOX-120a CCD camera $(640 \times 480$ pixels $)$ at a frame rate of $3.81 \mathrm{fps}$ with an exposure time of $250 \mathrm{~ms}$ and are available as supporting information at: http://web.mit.edu/soulages/www/MIT/Elastic_Instabilities.html.

\subsubsection{Comparison with results of numerical simulation}

A comparison between the SPTT model numerical predictions and the experimental streakline images is shown at different Weissenberg numbers in Fig. 12. The central difficulty that arises in quantitative comparisons of experimental measurements and single-mode numerical simulations of dilute polymer solutions is the modal distribution of the elastic contribution to the total viscoelastic stress. In a multimode computation with an $N$ bead-spring chain model, each mode $i=1,2, \ldots, N$ (each with progressively shorter relaxation time $\lambda_{1}>\lambda_{2}>\lambda_{3}>\cdots>\lambda_{N}$ ) makes a contribution $G_{i}=n k_{B} T$ to the total elastic modulus, and a contribution $\eta_{i}=n k_{B} T \lambda_{i}$ to the total viscosity. Any suitable measure of the mean relaxation time, for example $\bar{\lambda}=\Sigma_{i}\left(\eta_{i} \lambda_{i}\right) / \Sigma\left(\eta_{i}\right)$, is thus less than the longest relaxation time $\lambda_{1}$. The breadth of this distribution in the relaxation times is captured in "universal measures" such as the ratio $U_{\eta \lambda}=\Sigma_{i}\left(\lambda_{i}\right) / \lambda_{1}$ for the Rouse and Zimm models [67]. For a bead-spring chain in a theta solvent, the Zimm model with dominant hydrodynamic interactions gives $U_{\eta \lambda} \simeq 2.39$ [67,68]. By contrast, for any single mode dumbbell model the universal ratio is $U_{\eta \lambda}=1$ by definition, and all of the fluid elasticity is collapsed into the single viscoelastic relaxation mode.

This difference between single and multimode models is important if one seeks to compare the predictions of a single mode model with experimental data on a quantitative basis. If the relaxation time $\lambda_{1}$ is measured independently, then a computation with a single mode model over-estimates the total effects of viscoelasticity in a complex flow at moderate $W i$ (because in reality some of the shorter relaxation modes are "relaxed out" and should not contribute to the elastic stress). The longest relaxation time $\lambda_{1}$ in a dilute solution can be measured in CaBER experiments [69] whereas shear flow measurements of the steady shear viscosity and the first normal stress coefficient $\Psi_{1}$ (if measurable) can be used to evaluate a mean relaxation time $\bar{\lambda}=\Psi_{1} / 2 \eta_{P}[70,71]$. For highly viscous Boger fluids, it is possible to measure independently both $\Psi_{1}$ and $\lambda_{1}$ in a CaBER experiment and thus evaluate the breadth of the relaxation time spectrum directly; however, for low viscosity aqueous polymer solutions, the first normal stress difference is immeasurably small. When comparing experimental observations with computations, the choice must then be made as to whether to perform the calculation at the same value of Weissenberg number based on $\lambda_{1}$ or the mean relaxation time $\bar{\lambda}$. For a simple Zimmlike bead-spring model with dominant hydrodynamic interactions, we have $\lambda_{i} \simeq \lambda_{1} / i^{3 / 2}$ and $\Sigma_{i}\left(\lambda_{i}\right) \simeq \lambda_{1} \Sigma_{i}\left(1 / i^{3 / 2}\right)=\lambda_{1} \zeta(3 v)$, where $\zeta$ is the Riemann zeta function and $v$ is the solvent quality ( $v=0.5$ for a theta solvent). The universal ratio for this model is $U_{\eta \lambda} \simeq$ $\zeta(3 v)(\simeq 2.16$, considering a solvent quality exponent of $v \simeq 0.55$ for PEO in glycerol/water). The mean relaxation time determined from viscometric properties would then be $\bar{\lambda}=\Sigma_{i}\left(\eta_{i} \lambda_{i}\right) / \Sigma\left(\eta_{i}\right) \simeq$ $\lambda_{1} \zeta(6 v) / \zeta(3 v)=\lambda_{1} / 1.88$. Quantitative agreement with single mode computations should thus only be anticipated to within a factor of $\zeta(3 v) / \zeta(6 v) \approx 2$. In the following computations we use the value $\lambda_{1}$ because it is directly and independently measured through capillary thinning experiments (i.e. $\lambda_{1} \equiv \lambda_{\text {CaBER }}$ ); however we show that closer agreement between the critical conditions appears to be obtained if we compare experimental observations with a computation performed at $W i_{\text {num }}=\lambda_{1} \zeta(6 v) \dot{\gamma} / \zeta(3 v) \simeq \lambda_{\text {CaBER }} \dot{\gamma} / 2$.

The results in Fig. 12 are presented for both flow regimes: the symmetric Newtonian-like behavior (Fig. 12(a)) and the 3D timedependent flow (Fig. 12(c)). The SPTT model qualitatively captures the main differences between these two flows. The experimental streaklines and computed streamlines in Fig. 12(a) and (b) are symmetric, smooth and monotonically curved near the stagnation point. After incrementing the flow rate (or the Weissenberg number), the streamlines flatten in the stagnation region and the experimental flow becomes time-dependent and 3D beyond a critical Weissenberg number $W i_{\text {crit }} \simeq 3.2$ as shown in Fig. 12 (c). The single mode numerical computations predict a loss of flow sta- 


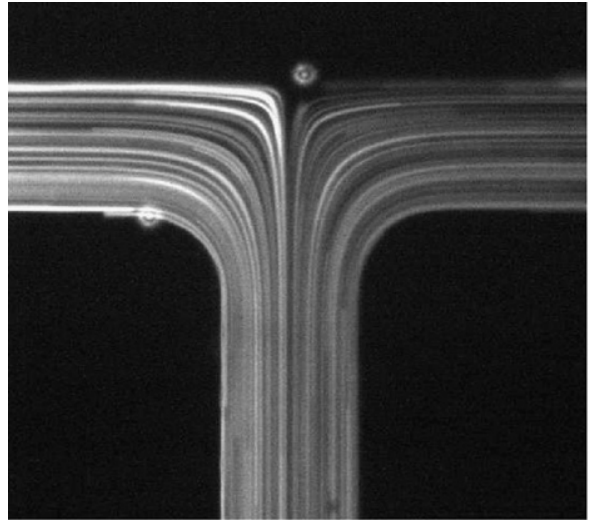

(a) $W i_{e x p}=2.35$

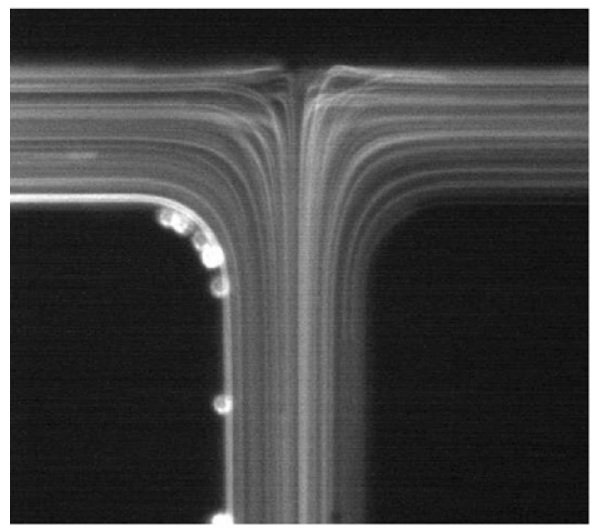

(c) $W i_{e x p}=4.69$

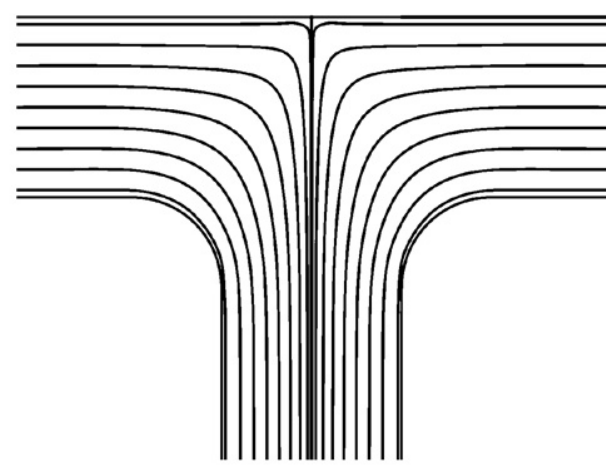

(b) $W i_{\text {num }}=1.17$

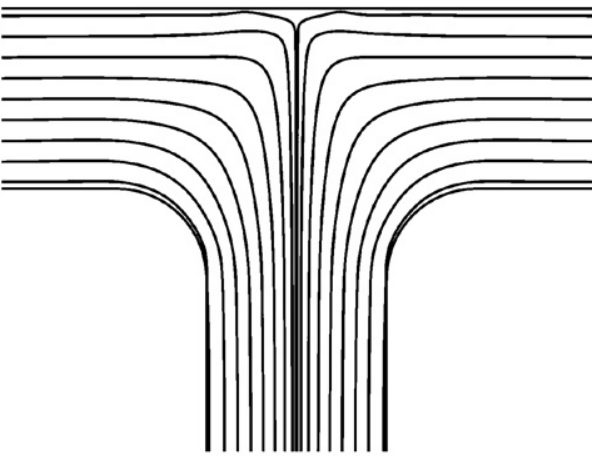

(d) $W i_{\text {num }}=2.35$

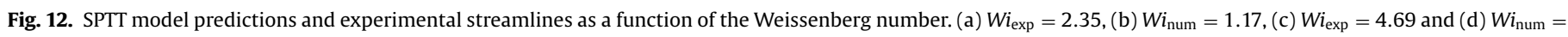
2.35

bility beyond a critical Weissenberg number of $W i_{\text {num }} \simeq 1.5$ and the streamlines shown in Fig. 12(d) are representative streamlines corresponding to an unsteady flow at one instance in time. This agreement is good, recognizing the difference and the limitations of a single mode simulation. In Fig. 12(c), the 3D character of the flow at high Weissenberg number is revealed by the crossing of fluid streaklines over the depth of measurement $\left(\delta z_{m}=14.5 \mu \mathrm{m}\right.$, representing about one third of the total channel depth). Although it is possible to compute microfluidic flows that capture such threedimensional features for Newtonian fluids [56], it is not yet viable to compute accurately three-dimensional time-dependent viscoelastic flows in reasonable CPU times.

In order to quantitatively assess the performance of the SPTT model, we superpose the numerically computed streamlines and experimental streaklines for symmetric flow conditions as shown in Fig. 13, once again using the conversion $\bar{\lambda}=\lambda_{\text {CaBER }} \zeta(6 v) / \zeta(3 v)$ so that $W i_{\text {num }}=W i_{\text {exp }} / 2$. The agreement between the model and the experimental data is quite satisfactory. It clearly indicates the ability of the model to accurately describe the global spatial characteristics of the viscoelastic flow in the T-shaped microchannel in the absence of a recirculating cavity.

Under identical experimental conditions (i.e. the same volumetric flow rates and same $R e$ and $W i$ numbers), we show the same comparison for the microchannel with a recirculating cavity in Fig. 14. The presence of the cavity leads to major differences in the kinematics and a loss in symmetry in the flow beyond a critical

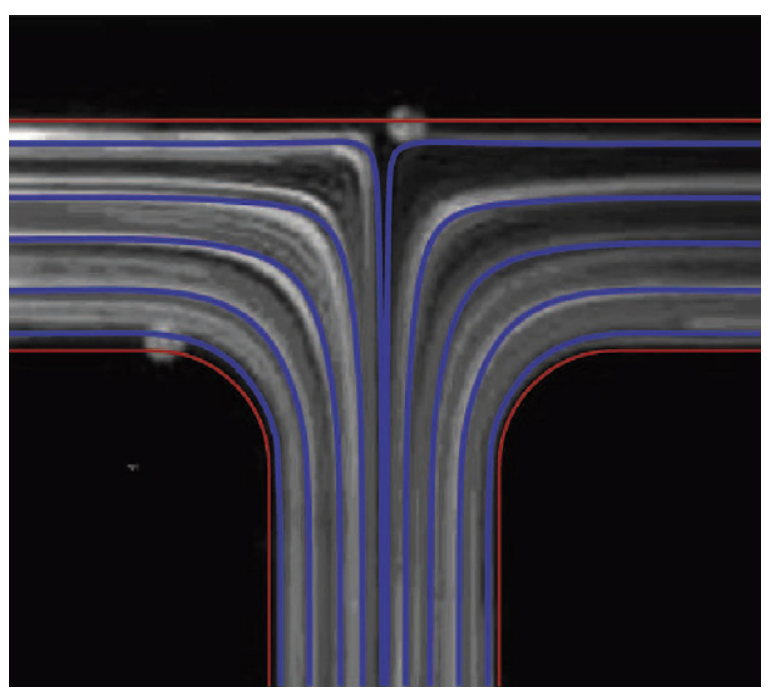

Fig. 13. Comparison of experimental streamline and SPTT model predictions (blue solid lines) for the microchannel without cavity: $Q=10 \mu \mathrm{L} / \mathrm{h}\left(W i_{\exp }=2.93 ; R e=\right.$ $3.41 \times 10^{-3}$ ). (For interpretation of the references to colour in this figure legend, the reader is referred to the web version of the article.) 


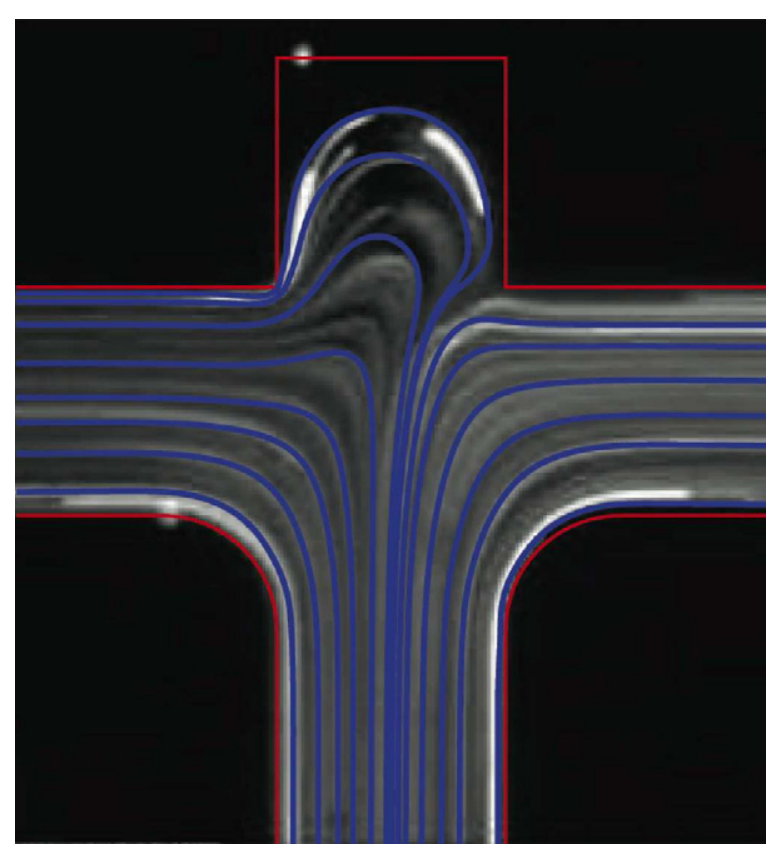

Fig. 14. Experimental streamline and SPTT model predictions (blue solid lines) for the microchannel with cavity using the same experimental conditions as in Fig. 13: $Q=10 \mu \mathrm{L} / \mathrm{h}\left(W i_{\exp }=2.93 ; R e=3.41 \times 10^{-3}\right)$. (For interpretation of the references to colour in this figure legend, the reader is referred to the web version of the article.)

flow rate. The stagnation point is not pinned on the microchannel walls anymore but is free to move. As a result, the dividing streamline $(x=0)$ is also unconstrained. The development of large tensile stresses in the region of planar extension illustrated in Fig. 9(a) together with curved streamlines leads to a symmetry-breaking bifurcation as shown in Fig. 14. Both experiments and calculations at lower $W i$ are symmetric (as detailed below). The flow transition is numerically observed for $W i=2 W i_{\text {num }} \simeq 2.5$, which is in good agreement with the value measured experimentally $(W i \simeq 2.4)$. The single mode SPTT model gives a very good quantitative description of the spatial characteristics of the steady fluid streamlines. In particular, the extent of the recirculating flow in the cavity is well captured by the model. Also, the local radius of curvature of the streamlines in the neighborhood of the cavity is accurately predicted by the numerical simulations.

In the following section, we analyze in more detail the different elastically driven flow transitions observed in the T-shaped microchannel with a recirculating cavity and compare them with the predictions of the single-mode SPTT model.

\subsection{Free stagnation point flow}

\subsubsection{Viscoelastic and Newtonian flow comparison}

A comparison of the streak-images obtained at different volumetric flow rates (i.e. different $W i$ numbers) for the viscoelastic dilute PEO solution and for the viscous glycerol/water solvent is shown in Fig. 15. The elasticity number for the viscoelastic solution is $E l=W i / R e=861$. In Fig. 15(a)-(e), the Reynolds number is less than $R e \leq 3.4 \times 10^{-2}$, so that we can neglect inertia and focus on the effect of elasticity on the stagnation flow. As we noted in Figs. 9 and 10, the presence of a recirculating flow in the cavity affects both the velocity and stress fields in the region of strong planar extension, which leads to noticeable differences in the fluid streamline patterns beyond a critical flow rate. The stagnation point is located on a free streamline, resulting in a non-zero velocity gradient at the origin of the laboratory frame.
By contrast to the flow in the channel without a cavity, we observe two distinct elastically driven flow transitions in Fig. 15(a)-(e). The flow first transitions from a steady symmetric Newtonian-like behavior (Fig. 15(a)) via a symmetry-breaking bifurcation to a steady asymmetric flow at a critical flow rate of $Q_{c} \simeq 8.0 \mu \mathrm{L} / \mathrm{h}$ (Fig. 15(b)). At higher flow rates, a second instability leads to a time-dependent 3D flow (Fig. 15(c)-(e)). These two transitions occur at a critical Weissenberg number $W i_{\text {crit } 1} \simeq 2.4$ and $W i_{\text {crit2 }} \simeq 5.3$, respectively. The second transition from steady 2D asymmetric flow to unsteady 3D flow occurs at a substantially higher critical Weissenberg number, namely $W i_{\text {crit2 }} \simeq 5.3$ compared to $W i_{\text {crit }} \simeq 3.2$ for the microchannel with pinned stagnation point due to the large stress gradients observed in this geometry (cf. Fig. 10).

As in the earlier studies of planar elongational flow in a cross-slot [30], it appears that the strand of highly oriented polymeric material in the region of planar extension leads to a symmetry-breaking bifurcation at $W i_{\text {crit } 1} \simeq 2.4$. The steady character of this first elastic instability was captured using digital video by imaging over several minutes at a frame rate of $3.81 \mathrm{fps}$ and an exposure time of $250 \mathrm{~ms}$. The corresponding movies can be found as supporting material at: http://web.mit.edu/soulages/www/MIT/Elastic_Instabilities.html. This flow asymmetry is very similar to that observed by Arratia et al. who investigated the cross-slot flow of a polyacrylamide viscoelastic solution [30]. In their study, the free stagnation point coupled to large tensile stresses led to a steady symmetry-breaking flow asymmetry for $W i \simeq 4.5$ and $R e \leq 10^{-2}$. The asymmetric flow shown in Fig. 15(b) is also bistable $[30,28]$ and the mirror image of the recirculating flow patterns can also be shown experimentally. Small random perturbations in the flow rate when approaching the critical Weissenberg number $W i_{\text {crit1 }}$ control the final direction of the flow in the cavity.

At higher flow rates corresponding to $W i_{\text {crit } 2} \simeq 5.3$, the flow transitions from a steady asymmetric bifurcation to a 3D timedependent flow as shown in Fig. 15(c)-(e). The 3D nature of the flow instability is again revealed by the crossing of the streaklines. As observed in the geometry without a cavity, the flow eventually becomes chaotic at high Weissenberg number, which is desirable for mixing purposes [65].

The corresponding Newtonian case shown in Fig. 15(f)-(j) is symmetric and stable for all the volumetric flow rates tested in this study $\left(R e \leq 6.5 \times 10^{-2}\right)$. For the highest flow rate $Q=100 \mu \mathrm{L} / \mathrm{h}$, a slight asymmetry is visible in the streamline pattern as shown in Fig. 15(j). This is due to small imbalances in the volumetric flow rates at the two channel inlets. Because of the large pressure gradients existing at high flow rates, some leakage between the Tygon tubing and the PDMS channel can occasionally be seen, which is responsible for the very small observed asymmetry at high flow rates. Numerical simulations confirm the negligible effect of inertia, and the predicted streamlines are symmetric and qualitatively similar to those predicted at lower flow rates.

In the next section, predictions of the single-mode SPTT model will be compared to experimental streak-images before and after the onset of the first (steady) flow transition observed in the free stagnation point flow $\left(W i_{\text {crit1 }} \simeq 2.4\right)$.

\subsubsection{Symmetric flow numerical simulation results}

The numerical predictions of the SPTT model and the experimental streak-images are shown in Fig. 16 for the symmetric case $\left(W i<W i_{\text {crit } 1} \simeq 2.4\right)$ at two different Weissenberg numbers. As in Section 4.3.2, the comparison is performed such that $W i_{\text {num }}=$ $\lambda_{1} \zeta(6 v) \dot{\gamma} / U_{\eta \lambda} \simeq W i_{\exp } / 2$. For both flow rates shown, the fluid streamlines are symmetric and the general evolution in the flow is well-captured by the SPTT model. When the Weissenberg number is increased, the magnitude of the first normal stress difference becomes larger in the region of strong planar extension. This 


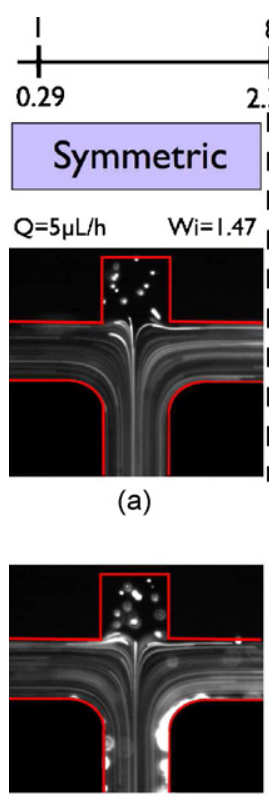

(f)

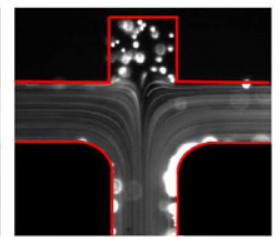

(g)

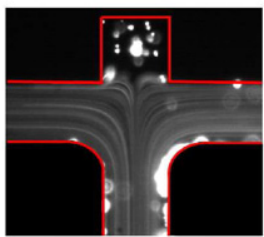

(h)

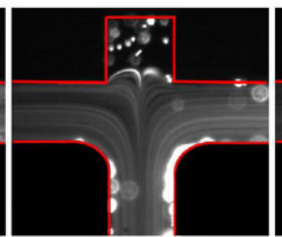

(i)

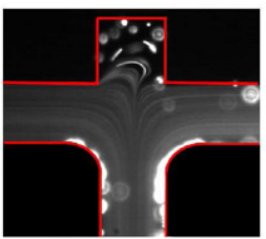

(j)

Fig. 15. Viscoelastic ((a)-(e)) and Newtonian ((f)-(j)) flow streamline patterns as a function of the volumetric flow rate $Q$.

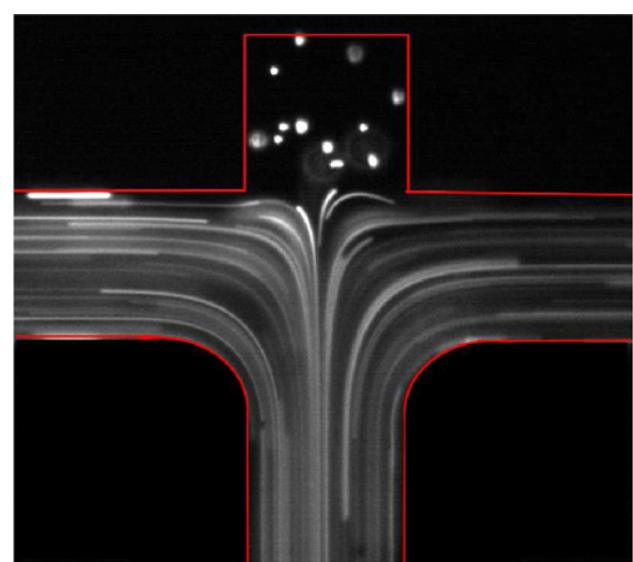

(a) $W i_{e x p}=1.17$

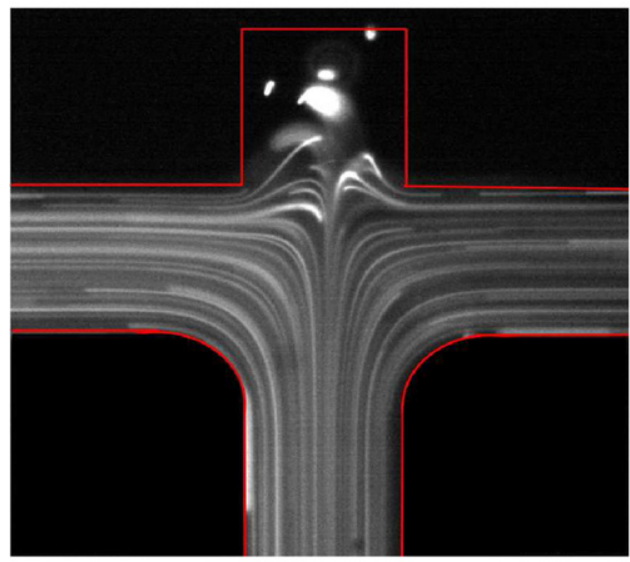

(c) $W i_{e x p}=2.05$

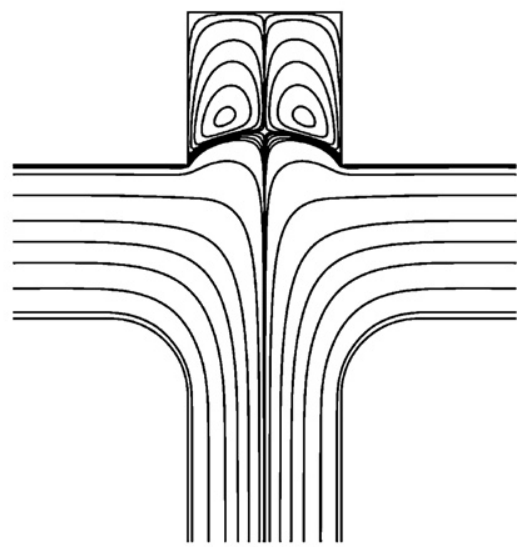

(b) $W i_{\text {num }}=0.59$

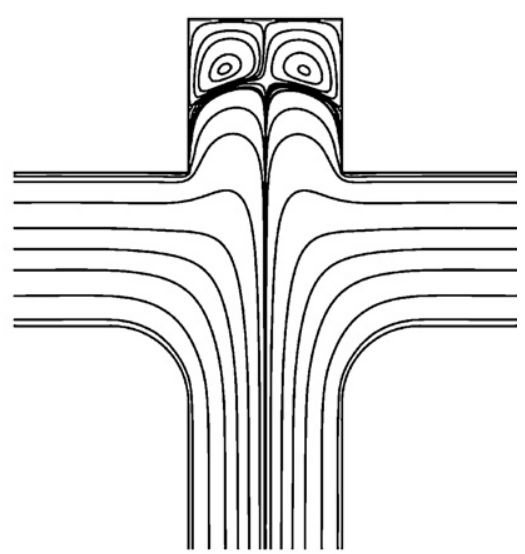

(d) $W i_{\text {num }}=1.17$

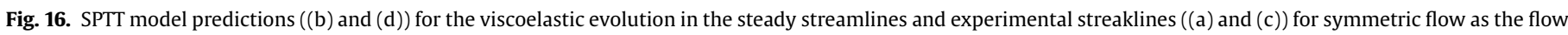
rate is increased. (a) $W i_{\exp }=1.17$, (b) $W i_{\text {num }}=0.59$, (c) $W i_{\exp }=2.05$ and (d) $W i_{\text {num }}=1.17$. 
increase in $N_{1}$ is responsible for the differences observed in the structure of the recirculating flow in the cavity observed in Fig. 16. It is important to note that the symmetric recirculating vortices in the cavity predicted in Fig. 16(b) and (d) are too weak to observe with the exposure time of $250 \mathrm{~ms}$ used for streakline imaging. Numerically, the recirculating flow in each recirculation only represents $9.5 \times 10^{-2}$ and $1.5 \times 10^{-2} \%$ of the total volumetric flow rate entering the T-channel through one arm for Fig. 16(b) and (d), respectively. The particles are displaced only slightly in this time and thus the corresponding streaklines could not be experimentally captured. However, the size of the secondary core-vortex flows (represented by dark regions with no streamlines in Fig. 16(a) and (c)) decreases with increasing Weissenberg numbers. As the level of elasticity increases, the tensile stresses in the flow lead to increasing penetration of the dividing streamline into the cavity. This is in good general agreement with numerical predictions as shown in Fig. 16(b) and (d).

\subsubsection{Symmetry-breaking bifurcation}

In Fig. 17, we compare the SPTT model predictions to the experimental streakline patterns observed after the onset of the first symmetry-breaking instability, namely for Weissenberg numbers between $W i_{\text {crit } 1} \simeq 2.4$ and $W i_{\text {crit } 2} \simeq 5$.3. The SPTT model accurately captures the experimental streak-images and the asymmetric pen- etration of the primary flow into the cavity. At high Weissenberg numbers, the large tensile stresses in the region of strong planar extension tend to "pull" the dividing streamline progressively outside of the cavity as observed both experimentally and numerically in Fig. 17. The small corner vortices predicted by the model in the quiescent (upper) corners of the cavity cannot be resolved experimentally due to the local small velocities with the current imaging system.

The absence of flow asymmetries for the Newtonian case in Fig. 15(g) together with the very low Reynolds numbers attained in this microfluidic geometry $\left(R e \leq 3.4 \times 10^{-2}\right)$ clearly indicates that this flow transition is driven by fluid elasticity. The large normal stress gradients in the region of strong planar extension shown in Fig. 9(a) together with curved streamlines are thus responsible for the observed symmetry-breaking bifurcation. To further illustrate the purely elastic character of this flow instability, corresponding creeping flow numerical simulations have also been done at nominally zero Reynolds number as shown in Fig. 18 by setting the inertial terms on the left hand side of Eq. (4) to zero. There is immeasurable difference between the two streamline patterns demonstrating that inertial effects are not responsible for the observed flow transition.

In order to document the characteristics of this symmetrybreaking bifurcation, we have measured the magnitude of the

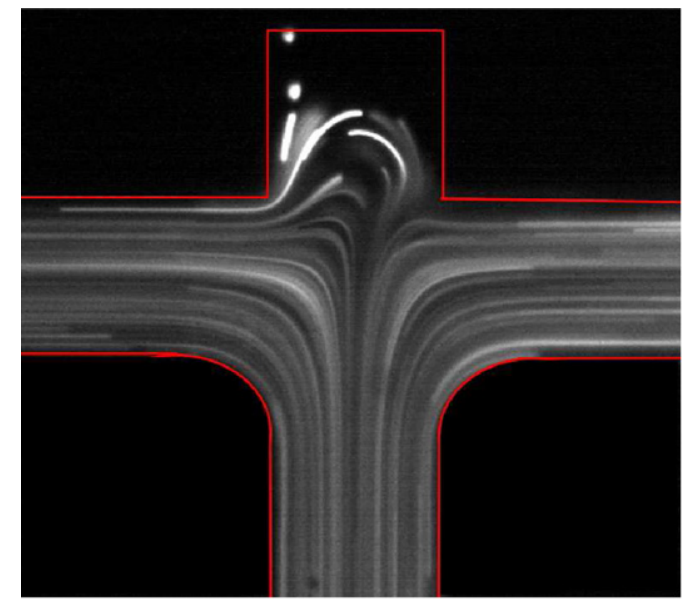

(a) $W i_{e x p}=2.64$

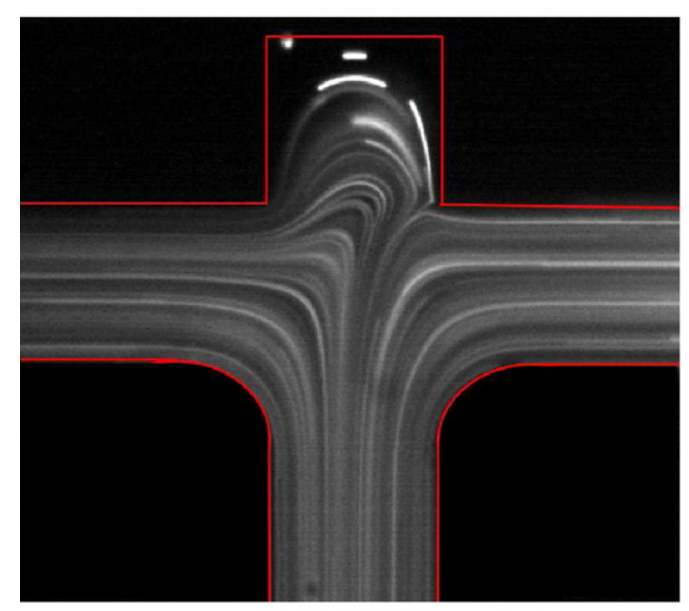

(c) $W i_{e x p}=3.52$

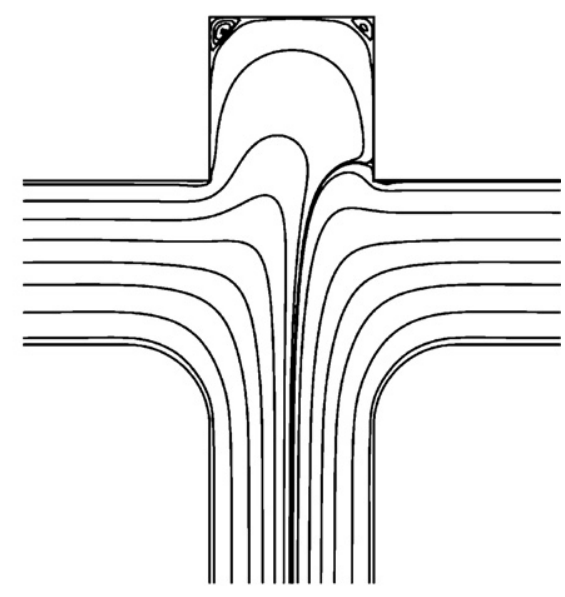

(b) $W i_{n u m}=1.32$

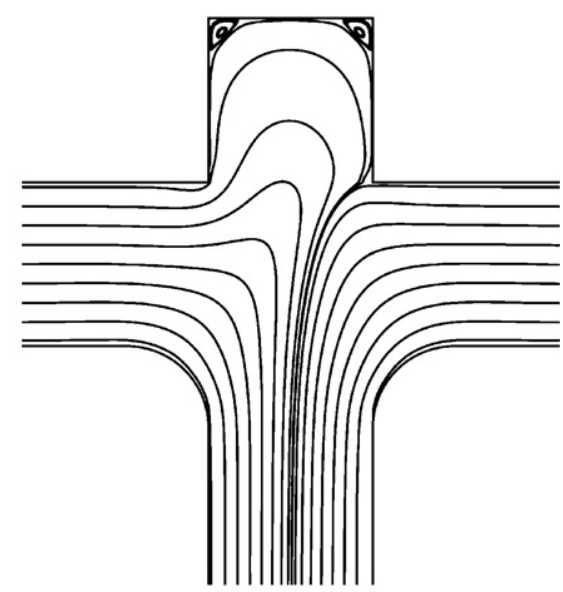

(d) $W i_{n u m}=1.76$

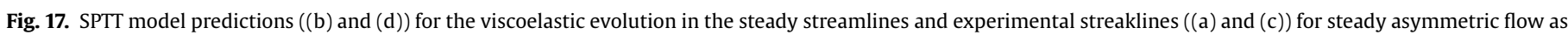
the flow rate is increased. (a) $W i_{\text {exp }}=2.64$, (b) $W i_{\text {num }}=1.32$, (c) $W i_{\text {exp }}=3.52$ and (d) $W i_{\text {num }}=1.76$. 
deviations from symmetric flow in the channel outlet, $90 \mu \mathrm{m}$ away from the origin as indicated by the horizontal dashed blue line in Fig. 19(a). For illustrative purposes, the dividing streamline has also been colored in black in the figure. Because of mass conservation, for each incoming stream entering the microchannel at a volumetric flow rate $Q$, the volumetric flow rate at the channel outlet is

$Q=\bar{V}_{1} h_{1} d=\bar{V}_{2} h_{2} d$,

where $d$ represents the (constant) microchannel depth, $\bar{V}_{i}$ is the average velocity for stream $i(i=1$ or 2$)$ and $h_{i}$ is the distance between the dividing streamline and the microchannel sidewalls measured along the $x$-axis as shown in Fig. 19(a) $\left(h=h_{1}+h_{2}\right.$, where $h$ is the total width of the outflow channel). The squared normalized velocity deviations from symmetric flow $(\Delta V / V)^{2}$ can thus be written as

$\left(\frac{\Delta V}{V}\right)^{2}=\left(\frac{\bar{V}_{2}-\bar{V}_{1}}{\bar{V}_{1}}\right)^{2}=\left(\frac{h_{1}}{h_{2}}-1\right)^{2}$.

According to Eq. (7), $(\Delta V / V)^{2}=0$ for a symmetric flow and $(\Delta V / V)^{2} \rightarrow 1$ for a fully asymmetric flow. Fig. 19(b) shows the squared normalized velocity deviations from symmetric flow as a function of the Weissenberg number together with numerical predictions at a distance of 50, 75 and $90 \mu \mathrm{m}$ away from the origin of the laboratory frame. The comparison is performed again such that $W i_{\text {num }}=\lambda_{1} \zeta(6 v) \dot{\gamma} / \zeta(3 v) \simeq W i_{\exp } / 2$. The results are shown for $W i \leq W i_{\text {crit2 }}$ and the maximum Weissenberg number for which convergent numerical results could be obtained is $W i_{\max }=4.4$.

For $W i<W i_{\text {crit } 1}$, the flow remains symmetric and $(\Delta V / V)^{2} \approx$ 0 (within experimental fluctuations). After the onset of the symmetry-breaking bifurcation, the magnitude of the asymmetry $(\Delta V / V)^{2}$ varies approximately linearly with $W i$, a typical behavior of supercritical bifurcations [72]. The 2D computations underestimate the magnitude of the deviations when the location of the transverse line is taken as $y=90 \mu \mathrm{m}$ or $y=75 \mu \mathrm{m}$. Much better agreement is found at a distance of $50 \mu \mathrm{m}$ from the origin of the laboratory frame.

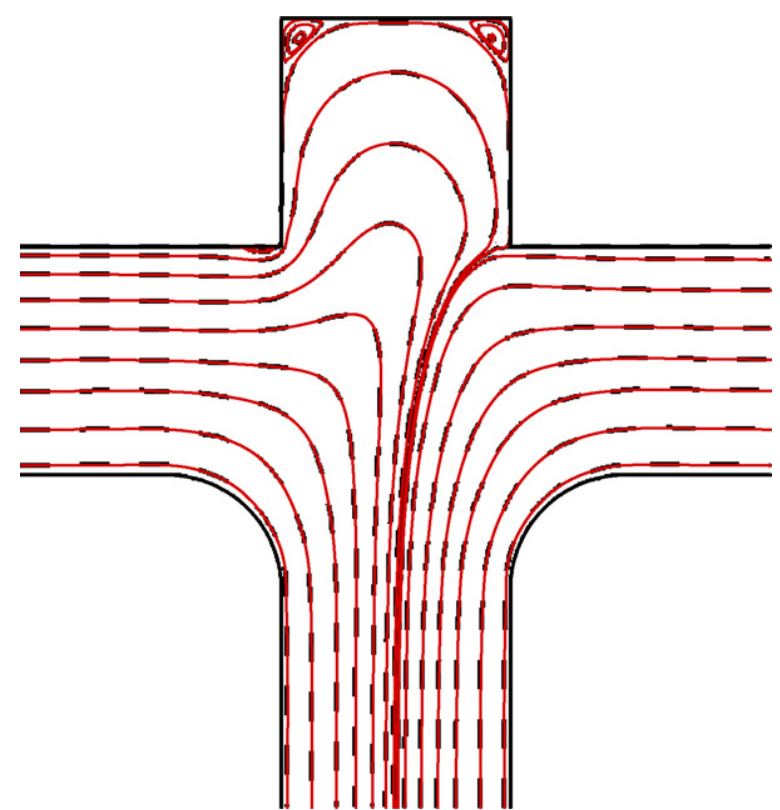

Fig. 18. Effect of inertia on the streamline patterns for the T-shaped microchannel with recirculating cavity $\left(\varepsilon=7.0 \times 10^{-6}\right)$. The solid red and dashed black streamlines correspond to finite inertia $\left(R e=2.05 \times 10^{-3}\right)$ and creeping $(R e=0)$ flow, respectively $(W i=1.76)$. (For interpretation of the references to colour in this figure legend, the reader is referred to the web version of the article.)

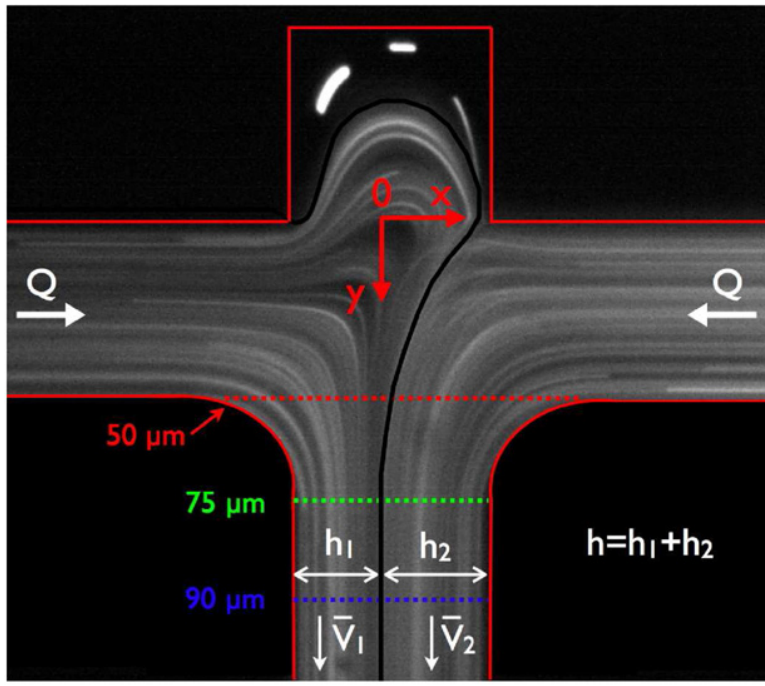

(a) Geometrical details.

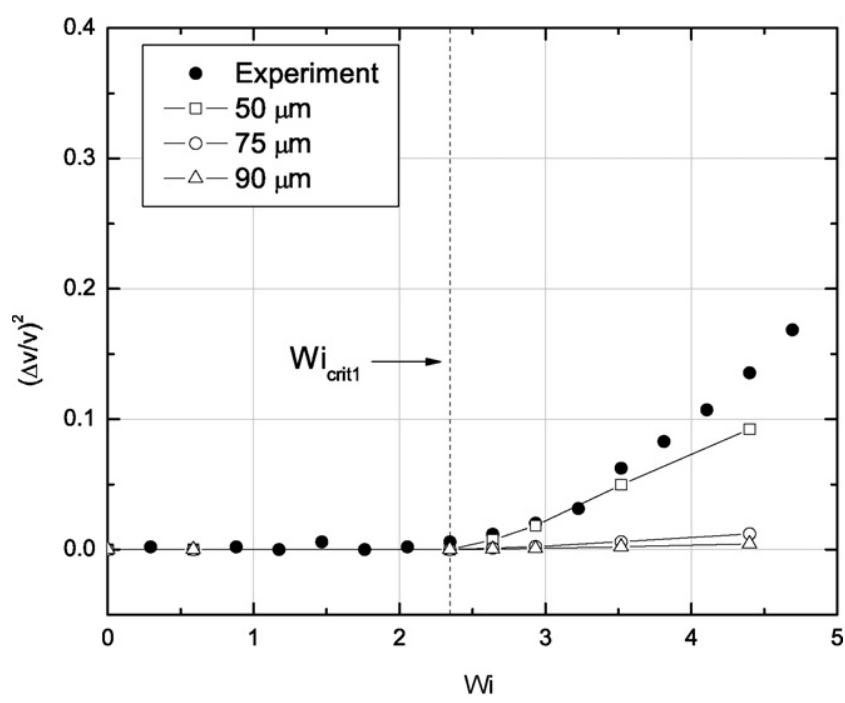

(b) Normalized velocity deviations.

Fig. 19. (a) Geometrical details and (b) normalized velocity deviations from symmetric flow (filled symbols) in the microchannel outlet ( $90 \mu \mathrm{m}$ away from S.P.) together with numerical predictions (open symbols) at a distance of 50, 75 and $90 \mu \mathrm{m}$ away from the S.P. The critical Weissenberg number at the onset of steady asymmetric flow is also indicated by a vertical dashed solid line in the figure $\left(W i_{\text {crit1 }}=2.4\right)$. The results are shown for $W i \leq W i_{\text {crit2 }}$ and the maximum Weissenberg number for which numerical results are plotted is $W i_{\max }=4.4$.

This discrepancy in the strength of the flow asymmetry at a given value of $W i=W i_{\text {crit } 1}>0$ may be a consequence of the finite depth of the T-channel geometry $(d=50 \mu \mathrm{m})$ which gives rise to three-dimensionality in the local experimental flow that cannot be captured by the 2D simulations.

The observations and calculations strongly suggest that the observed bifurcation is a supercritical steady viscoelastic flow transition with strong similarities to the cross-slot observations of Arratia et al. [30] that were duplicated numerically by Poole and coworkers [35].

\section{Conclusion and outlook}

In this study, we have investigated the structure and stability of steady planar stagnation flows of a dilute viscoelastic PEO solution using two different T-shaped microchannels, with and without, a recirculating cavity region, respectively. We have shown that 
the kinematic differences near the stagnation point in these two geometries control the magnitude of the large tensile normal stress differences in the vicinity of the stagnation point as well as the critical conditions and spatiotemporal dynamics of the resulting elastically driven flow asymmetries. For the free stagnation point flow, a strand of highly oriented polymeric material is formed in the region of strong planar extensional flow, which results in an additional symmetry-breaking transition at intermediate Weissenberg numbers. For each stagnation flow, we also observed a flow transition from a steady to a three-dimensional time-dependent flow at a critical Weissenberg number. The critical conditions are substantially lower for the pinned stagnation point flow.

The spatial characteristics of these purely elastic flow instabilities were compared with two-dimensional numerical predictions using a single-mode simplified Phan-Thien-Tanner (SPTT) model. The calculations were shown to quantitatively capture the evolution in the streamline patterns with increasing Weissenberg number as well as predict the onset of a steady 2D asymmetric flow beyond a critical flow rate. Idealized creeping flow calculations with no fluid inertia demonstrate the purely elastic nature of the different flow transitions.

Future work will involve a detailed analysis of the local velocity field in the vicinity of the stagnation point using microparticle image velocimetry to further document the nature of the bifurcation. We also hope to make use of other viscoelastic fluids such as wormlike micellar solutions to investigate the role of the magnitude of strain-hardening in the extensional viscosity on the local stresses near the stagnation point and the corresponding flow stability.

\section{Acknowledgments}

This work made use of facilities in the MIT Microelectronics Technology Laboratory and was supported by the Swiss National Science Foundation (Grant PBEZ2-115179). The authors acknowledge the funding support provided by Fundação para a Ciência e a Tecnologia under projects PTDC/EQU-FTT/71800/2006 and PTDC/EQU-FTT/70727/2006.

\section{References}

[1] H.A. Stone, S. Kim, Microfluidics: basic issues, applications, and challenges, AIChE Journal 47 (6) (2001) 1250-1254.

[2] G.M. Whitesides, The origins and the future of microfluidics, Nature 442 (7101) (2006) 368-373.

[3] P. Gravesen, J. Branebjerg, O.S. Jensen, Microfluidics-a review, Journal of Micromechanics and Microengineering 3 (1993) 168-182.

[4] T. Pfohl, F. Mugele, R. Seemann, S. Herminghaus, Trends in microfluidics with complex fluids, ChemPhysChem 4 (12) (2003) 1291-1298.

[5] T.M. Squires, S.R. Quake, Microfluidics: fluid physics at the nanoliter scale, Reviews of Modern Physics 77 (3) (2005) 977-1026.

[6] D.J. Beebe, G.A. Mensing, G.M. Walker, Physics and applications of microfluidics in biology, Annual Review of Biomedical Engineering 4 (2002) 261-286.

[7] C. Hansen, S.R. Quake, Microfluidics in structural biology: smaller, faster... better, Current Opinion in Structural Biology 13 (5) (2003) 538-544.

[8] C.J. Pipe, G.H. McKinley, Microfluidic rheometry, Mechanics Research Communications 36 (2009) 110-120.

[9] P. Pakdel, G.H. McKinley, Elastic instability and curved streamlines, Physical Review Letters 77 (12) (1996) 2459-2462.

[10] A. Öztekin, B. Alakus, G.H. McKinley, Stability of planar stagnation flow of a highly viscoelastic fluid, Journal of Non-Newtonian Fluid Mechanics 72 (1) (1997) 1-29.

[11] W.B. Zimmerman, J.M. Rees, T.J. Craven, Rheometry of non-Newtonian electrokinetic flow in a microchannel T-junction, Microfluid Nanofluid 2 (2006) 481-492.

[12] K. Walters, R.I. Tanner, The motion of a sphere through an elastic liquid, in: R.P. Chhabra, D. De Kee (Eds.), Transport Processes in Bubbles, Drops and Particles, Hemisphere Publ. Corp., New York, 1992, pp. 73-86.

[13] G.H. McKinley, Steady and transient motion of spherical particles in viscoelastic liquids, in: R.P. Chhabra, D. De Kee (Eds.), Transport Processes in Bubbles, Drops and Particles, Taylor and Francis, New York, 2002, pp. 338-375, Chapter 14

[14] C. Bisgaard, Velocity fields around spheres and bubbles investigated by Laser-Doppler anemometry, Journal of Non-Newtonian Fluid Mechanics 12 (3) (1983) 283-302.
[15] R. You, A. Borhan, H. Haj-Hariri, A finite volume formulation for simulating drop motion in a viscoelastic two-phase system, Journal of Non-Newtonian Fluid Mechanics 153 (2-3) (2008) 109-129.

[16] F. Pan, A. Acrivos, Steady flows in rectangular cavities, Journal of Fluid Mechanics 28 (1967) 643.

[17] S. Taneda, Visualization of separating Stokes flows, Journal of the Physical Society of Japan 46 (6) (1979) 1935-1942.

[18] M.G.N. Perera, K. Walters, Long-range memory effects in flows involving abrupt changes in geometry. Part I: Flows associated with L-shaped and Tshaped geometries, Journal of Non-Newtonian Fluid Mechanics 2 (1) (1977) 49-81.

[19] T. Nishimura, K. Nakamura, A. Horikawa, Two-dimensional viscoelastic flow of polymer solution at channel junction and branch. Part I: T-shaped junction flow, Journal of the Textile Machinery Society of Japan 31 (1) (1985) 1-6.

[20] D.M. Binding, K. Walters, J. Dheur, M.J. Crochet, Interfacial effects in the flow of viscous and elastoviscous liquids, Philosophical Transactions of the Royal Society of London Series A-Mathematical Physical and Engineering Sciences 323 (1573) (1987) 449.

[21] L.E. Rodd, T.P. Scott, D.V. Boger, J.J. Cooper-White, G.H. McKinley, The inertioelastic planar entry flow of low-viscosity elastic fluids in micro-fabricated geometries, Journal of Non-Newtonian Fluid Mechanics 129 (1) (2005) 1-22.

[22] L.E. Rodd, J.J. Cooper-White, D.V. Boger, G.H. McKinley, Role of the elasticity number in the entry flow of dilute polymer solutions in micro-fabricated contraction geometries, Journal of Non-Newtonian Fluid Mechanics 143 (2-3) (2007) 170-191.

[23] E.S.G. Shaqfeh, S.J. Muller, R.G. Larson, The effects of gap width and dilutesolution properties on the viscoelastic Taylor Couette instability, Journal of Fluid Mechanics 235 (1992) 285-317.

[24] G.H. McKinley, P. Pakdel, A. Oztekin, Rheological and geometric scaling of purely elastic flow instabilities, Journal of Non-Newtonian Fluid Mechanics 67 (1996) 19-47.

[25] A. Groisman, V. Steinberg, Efficient mixing at low Reynolds numbers using polymer additives, Nature 410 (6831) (2001) 905-908.

[26] S. Gulati, D. Liepmann, S.J. Muller, Elastic secondary flows of semidilute DNA solutions in abrupt $90^{\circ}$ microbends, Physical Review E 78 (3) (2008) 036314.

[27] S. Gulati, S.J. Muller, D. Liepmann, Direct measurements of viscoelastic flows of DNA in a 2:1 abrupt planar micro-contraction, Journal of Non-Newtonian Fluid Mechanics 155 (1-2) (2008) 51-66.

[28] A. Groisman, M. Enzelberger, S.R. Quake, Microfluidic memory and control devices, Science 300 (5621) (2003) 955-958.

[29] A. Groisman, S.R. Quake, A microfluidic rectifier: anisotropic flow resistance at low reynolds numbers, Physical Review Letters 92 (9) (2004) 0945011-0945014-0945014.

[30] P.E. Arratia, C.C. Thomas, J. Diorio, J.P. Gollub, Elastic instabilities of polymer solutions in cross-channel flow, Physical Review Letters 96 (14) (2006) 144502

[31] E.F. Humphrey, D.H. Tarumoto, Fluidics, Fluid Amplifier Associates, Boston, 1965.

[32] K. Foster, G.A. Parker, Fluidics: Components and Circuits, Wiley Interscience, London, 1970.

[33] J.A. Odell, S.P. Carrington, Polymer Solutions in Strong Stagnation Point Extensional Flows, in: Flexible Polymer Chains in Elongational Flow: Theory and Experiment, Springer-Verlag, Berlin, 1999.

[34] J.A. Pathak, S.D. Hudson, Rheo-optics of equilibrium polymer solutions: wormlike micelles in elongational flow in a microfluidic cross-slot, Macromolecules 39 (25) (2006) 8782-8792.

[35] R.J. Poole, M.A. Alves, P.J. Oliveira, Purely elastic flow asymmetries, Physical Review Letters 99 (16) (2007) 164503.

[36] N.-T. Nguyen, S.T. Wereley, Fundamentals and Applications of Microfluidics, Artech House, 2002.

[37] D.C. Duffy, J.C. McDonald, O.J.A. Schueller, G.M. Whitesides, Rapid prototyping of microfluidic systems in poly(dimethylsiloxane), Analytical Chemistry 70 (23) (1998) 4974-4984.

[38] Y.N. Xia, G.M. Whitesides, Soft lithography, Annual Review of Materials Science 28 (1998) 153-184.

[39] J.C. McDonald, D.C. Duffy, J.R. Anderson, D.T. Chiu, H.K. Wu, O.J.A. Schueller, G.M. Whitesides, Fabrication of microfluidic systems in poly(dimethylsiloxane), Electrophoresis 21 (1) (2000) 27-40.

[40] J.M.K. Ng, I. Gitlin, A.D. Stroock, G.M. Whitesides, Components for integrated poly(dimethylsiloxane) microfluidic systems, Electrophoresis 23 (20) (2002) 3461-3473.

[41] T.P. Scott, Contraction/expansion flow of dilute elastic solutions in microchannels, M.S. Thesis, MIT, 2004

[42] R.G. Larson, Instabilities in viscoelastic flows, Rheologica Acta 31 (3) (1992) 213-263.

[43] G.H. McKinley, A. Tripathi, How to extract the Newtonian viscosity from capillary breakup measurements in a filament rheometer, Journal of Rheology 44 (3) (2000) 653-670.

[44] S.L. Anna, G.H. McKinley, Elasto-capillary thinning and breakup of model elastic liquids, Journal of Rheology 45 (1) (2001) 115-138.

[45] L.E. Rodd, T.P. Scott, J.J. Cooper-White, G.H. McKinley, Capillary break-up rheometry of low-viscosity elastic fluids, Applied Rheology 15 (1) (2005) 12-27.

[46] N. Phan-Thien, R.I. Tanner, New constitutive equation derived from network theory, Journal of Non-Newtonian Fluid Mechanics 2 (4) (1977) 353-365.

[47] C. Clasen, J.P. Plog, W.M. Kulicke, M. Owens, C. Macosko, L.E. Scriven, M. Verani, G.H. McKinley, How dilute are dilute solutions in extensional flows? Journal of Rheology 50 (6) (2006) 849-881. 
[48] V. Tirtaatmadja, G.H. McKinley, J.J. Cooper-White, Drop formation and breakup of low viscosity elastic fluids: Effects of molecular weight and concentration, Physics of Fluids 18 (4) (2006) 043101.

[49] W.W. Graessley, Polymer chain dimensions and the dependence of viscoelastic properties on concentration, molecular weight and solvent power, Polymer 21 (3) (1980) 258-262.

[50] M.S.N. Oliveira, M.A. Alves, F.T. Pinho, G.H. McKinley, Viscous flow through microfabricated hyperbolic contractions, Experiments in Fluids 43 (2-3) (2007) 437-451.

[51] P.J. Oliveira, F.T. Pinho, G.A. Pinto, Numerical simulation of non-linear elastic flows with a general collocated finite-volume method, Journal of NonNewtonian Fluid Mechanics 79 (1) (1998) 1-43.

[52] P.J. Oliveira, F.T. Pinho, Numerical procedure for the computation of fluid flow with arbitrary stress-strain relationships, Numerical Heat Transfer Part BFundamentals 35 (3) (1999) 295-315.

[53] M.A. Alves, P.J. Oliveira, F.T. Pinho, Benchmark solutions for the flow of Oldroyd$B$ and PTT fluids in planar contractions, Journal of Non-Newtonian Fluid Mechanics 110 (1) (2003) 45-75.

[54] P.J. Oliveira, F.T. Pinho, Plane contraction flows of upper convected Maxwell and Phan-Thien-Tanner fluids as predicted by a finite-volume method, Journal of Non-Newtonian Fluid Mechanics 88 (1-2) (1999) 63-88.

[55] M.S.N. Oliveira, P.J. Oliveira, F.T. Pinho, M.A. Alves, Effect of contraction ratio upon viscoelastic flow in contractions: The axisymmetric case, Journal of NonNewtonian Fluid Mechanics 147 (1-2) (2007) 92-108.

[56] M.S.N. Oliveira, L.E. Rodd, G.H. McKinley, M.A. Alves, Simulations of extensional flow in microrheometric devices, Microfluidics Nanofluidics 5 (2008) 809-826.

[57] M.A. Alves, P.J. Oliveira, F.T. Pinho, A convergent and universally bounded interpolation scheme for the treatment of advection, International Journal for Numerical Methods in Fluids 41 (1) (2003) 47-75.

[58] R.G. Larson, Constitutive Equations for Polymer Melts and Solutions, Butterworth Publishers, 1988.

[59] R. Fattal, R. Kupferman, Constitutive laws for the matrix-logarithm of the conformation tensor, Journal of Non-Newtonian Fluid Mechanics 123 (2-3) (2004) 281-285.

[60] A.M. Afonso, P.J. Oliveira, F.T. Pinho, M.A. Alves, The log conformation tensor approach in the finite volume method framework, Journal of Non-Newtonian Fluid Mechanics 157 (1-2) (2009) 55-65.
[61] O.G. Harlen, High-Deborah-number flow of a dilute polymer solution past a sphere falling along the axis of a cylindrical tube, Journal of Non-Newtonian Fluid Mechanics 37 (2-3) (1990).

[62] M.A. Alves, F.T. Pinho, P.J. Oliveira, The flow of viscoelastic fluids past a cylinder: finite-volume high-resolution methods, Journal of Non-Newtonian Fluid Mechanics 97 (2-3) (2001) 207-232.

[63] M.A. Hulsen, R. Fattal, R. Kupferman, Flow of viscoelastic fluids past a cylinder at high Weissenberg number: Stabilized simulations using matrix logarithms, Journal of Non-Newtonian Fluid Mechanics 127 (1) (2005) 27-39.

[64] G.H. McKinley, R.C. Armstrong, R.A. Brown, The wake instability in viscoelastic flow past confined circular-cylinders, Philosophical Transactions of the Royal Society of London Series A-Mathematical Physical and Engineering Sciences 344 (1671) (1993) 265-304.

[65] J.M. Ottino, S. Wiggins, Applied physics-designing optimal micromixers, Science 305 (5683) (2004) 485-486.

[66] P. Becherer, W. Saarloos, A.N. Morozov, Stress singularities and the formation of birefringent strands in stagnation flows of dilute polymer solutions, Journal of Non-Newtonian Fluid Mechanics 157 (1-2) (2009) 126-132.

[67] H.C. Öttinger, Stochastic Processes in Polymeric Fluids: Tools and Examples for Developing Simulation Algorithms, Springer-Verlag, Berlin, 1996.

[68] M. Kröger, A. Alba-Perez, M. Laso, H.C. Öttinger, Variance reduced Brownian simulation of a bead-spring chain under steady shear flow considering hydrodynamic interaction effects, Journal of Chemical Physics 113 (11) (2000) 4767-4773.

[69] V.M. Entov, E.J. Hinch, Effect of a spectrum of relaxation times on the capillary thinning of a filament of elastic liquid, Journal of Non-Newtonian Fluid Mechanics 72 (1) (1997) 31-53.

[70] J.P. Rothstein, G.H. McKinley, Non-isothermal modification of purely elastic flow instabilities in torsional flows of polymeric fluids, Physics of Fluids 13 (2) (2001) 382-396.

[71] I. Ghosh, G.H. McKinley, R.A. Brown, R.C. Armstrong, Deficiencies of FENE dumbbell models in describing the rapid stretching of dilute polymer solutions, Journal of Rheology 45 (3) (2001) 721-758.

[72] G. Iooss, D. Joseph, Elementary Stability and Bifurcation Theory, SpringerVerlag, Berlin, 1980. 